\title{
Residual stresses in gas-assisted injection molding
}

\author{
Frederico J. M. F. Custódio - Patrick D. Anderson • \\ Gerrit W. M. Peters • António M. Cunha • \\ Han E. H. Meijer
}

Received: 2 June 2009 / Accepted: 19 September 2009 / Published online: 6 October 2009

(C) The Author(s)2009. This article is published with open access at Springerlink.com

\begin{abstract}
Residual stresses are a major issue in the mechanical and optical behavior of injection-molded parts. In this study, we analyze their development in the case of gas-assisted injection molding (GAIM) of amorphous polymers. Flow-induced residual stresses are computed within a decoupled approach, in which elastic effects are neglected in the momentum balance, assuming a generalized Newtonian material behavior. In a staggered procedure, the computed viscous flow kinematics are used to calculate normal stresses employing a compressible version of the Rolie-Poly model. For the computation of thermally and pressure-induced residual stresses, a linear thermo-viscoelastic model is used. A 3-D finite element model for GAIM is employed, which is able to capture the kinematics of the flow front and whose capabilities to predict the thickness of the residual material layer have been validated by Haagh and Van de Vosse (Int J Numer Methods Fluids 28:1355-1369, 1998). In order to establish a clear comparison, the development of residual stresses is analyzed using standard injection molding and GAIM for a test geometry.
\end{abstract}

F. J. M. F. Custódio · P. D. Anderson ( $₫)$.

G. W. M. Peters · H. E. H. Meijer

Materials Technology, Eindhoven University of Technology,

P.O. Box 513, 5600MB, Eindhoven, The Netherlands

e-mail: p.d.anderson@tue.nl

A. M. Cunha

University of Minho, Braga, Portugal
Keywords Gas-assisted injection molding • Viscoelasticity • Compressible Rolie-Poly model • Flow-induced residual stresses - Thermally and pressure-induced residual stresses . Amorphous polymers $\cdot$ Numerical analysis

\section{Introduction}

Residual stresses in injection molding are responsible for the dimensional stability of molded parts and the anisotropy of their properties, i.e., mechanical and optical. There are mainly two sources of residual stresses (Baaijens 1991; Meijer 1997). The first, flow-induced stresses, are viscoelastic in nature and originate from the resistance of molecules to attain a preferential alignment with the flow direction (entropy driven). The second, the so-called thermally and pressure-induced stresses, originate from differential shrinkage induced by the combined effect of inhomogeneous cooling and pressure.

Upon processing, polymer molecules in the melt become aligned within the flow direction, attaining a degree of orientation that is dependent on the strain rate and on the relaxation times of individual molecules. However, once molecules become orientated, stresses in the fluid start to develop, a phenomena usually depicted by an increase of the first normal stress difference. Such stress development is related to the decrease in entropy of the molecules. Frozenin flow-induced stresses are known to dictate the longterm dimensional stability of injection-molded parts. Throughout the lifetime of a molded part, relaxation processes that yield shrinkage take place as oriented 
molecules strive to reach for more favorable conformations. However, since relaxation times of molecules become extremely high upon cooling through the glass transition temperature, $T_{\mathrm{g}}$, typically such processes take place over long times. As was shown by Caspers (1995), the resulting shrinkage depends on the molecular orientation. Furthermore, frozen-in orientation also introduces anisotropy of physical properties, e.g., mechanical, optical, and thermal. In addition to this, as was pointed out by Struik (1978), flow-induced stresses also affect the thermal expansivity of polymers below $T_{\mathrm{g}}$, as they result in a negative contribution to the reversible coefficient of thermal expansion.

Differential shrinkage in injection molded parts originates from the combined effect of inhomogeneous cooling and pressure. To understand how both mechanisms contribute to the final residual stresses, it is worth to first consider the case of free quenching, in which only thermal stresses develop. Upon quenching a flat sheet of an amorphous polymer, cooling becomes inhomogeneous since material near the surface cools first and faster. Hence, each material point across the sheet thickness solidifies at a different time. As the material layers near the surface solidify and stiffen, they shrink due to thermal contraction, imposing a compressive stress on the molten material in the core region that relaxes fast by viscous deformation. Later, when the core region starts to solidify, shrinkage thereof is prevented by layers previously solidified at the surface. As a consequence, the outer layers are put under compression while the core puts itself under tension upon shrinkage during cooling. Obviously, the residual stress profile depends on the combined effect of inhomogeneous cooling and changes in the elastic properties, in particular the modulus, with temperature and time. For injection-molding parts, which is the case of interest in the present study, the situation is more complex since solidification occurs in the presence of pressure that is only released upon opening of the mold. Due to differences in compressibility between the solidified layers close to the mold walls and the material which is undergoing vitrification, a variation of the pressure level upon cooling leads to differences in residual stresses. Hence, the changes in density of each material point are locally defined by the respective cooling and pressure history. Hastenberg et al. (1998) measured the residual stress profile of amorphous polymers in injection-molded flat plates using a modified removal layer technique. In contrast to free-quenching conditions, the authors measured significant tensile stresses near the surface, followed by a compressive transition region and a core under tensile stresses. Such a profile, and remarkably the presence of tensile stresses at the surface, is believed to be responsible for mechanical failure phenomena referred to as environmental stress cracking, see Mandell et al. (1981). Following the conclusions of Zoetelief et al. (1996), the residual stress profile in injection molding products is mostly determined by pressure, and not by differences in time of thermal contraction across the part's thickness. The situation for semi-crystalline materials is somewhat different. As was shown by Kamal et al. (1988), the residual stress profiles for high-density polyethylene (HDPE) are different from those of polystyrene (PS) and polycarbonate (PC). More specifically, compressive stresses were found to develop in the core region that increase to tensile stresses towards the walls.

It has been reported (Baaijens 1991; Douven 1991; Zoetelief et al. 1996; Kamal et al. 1988) that the magnitude of flow-induced stresses is about one to two orders lower than the magnitude of thermally and pressureinduced stresses. However, Wimberger-Friedl et al. (2003) showed, by comparing PS with PC, that the ratio between these two contributions to the final residual stresses depends on the polymer. Notably, the magnitude of flow-induced stresses is much higher for PS than for PC. The lower molecular weight and relaxation times of PC chains, when compared with PS, make of $\mathrm{PC}$ a difficult material to orient, thus enabling its use in optical applications that require low birefringence like CDs and DVDs. However, the contribution of thermally and pressure-induced stresses to the total residual stresses is supposed to be higher for PC instead, since these stresses scale with the material modulus. The authors in Wimberger-Friedl et al. (2003) conclude that both flow-induced and thermally and pressure-induced stresses induce, in equal magnitude, the birefringence of PC injection-molded parts.

Even though some research was devoted to the computation of residual stresses in injection molding, there has not yet been a study devoted to understanding how residual stresses develop in gas-assisted injection molding. Given the vast use of gas-assisted injection molding (GAIM) in polymer injection, the need for such type of studies motivates our investigation. The GAIM technology is mostly used to produce parts with hollow sections, structural ribs, bosses, or parts with regions with significant changes in thickness. Even though there are some variants, typically the GAIM process evolves with the following steps: first, polymer is injected until the mold cavity becomes partly filled, next (or during the polymer injection), the gas injection phase takes place, which is done in two phases: a gas penetration phase, during which the cavity walls are wet by the polymer melt, and a secondary penetration phase in which gas, under pressure control, holds the polymer part under 
pressure while cooling takes place. In the end, the gas is vented and the part ejected. Some of the advantages of this technology are the decrease of the part's weight, cycle time, injection pressure, clamping force, shrinkage, and warpage. Until recently, the numerical studies reported on GAIM have mainly focused on the prediction of the gas bubble shape and thickness of the residual polymer layer as a function of process variables, i.e., shot weight, gas pressure, etc. (Haagh and Van de Vosse 2001; Li et al. 2004; Parvez et al. 2002; Polynkin et al. 2005). Given the vast industrial application and high potential of GAIM, it is essential to also focus numerical studies on the prediction of properties. In this work, we propose a method for the computation of residual stresses in GAIM. We extend the finite element 3-D model developed by Haagh and Van de Vosse (1998) for GAIM and follow a decoupled approach in which the velocity-pressure problem is solved independently from the stress problem. Hence, at every computational time step, we compute the flow kinematics assuming a generalized Newtonian fluid behavior and plug them into a viscoelastic model to compute residual stresses. Such a staggered-scheme approach was validated by Baaijens (1991), where it was shown that minor differences in terms of normal stresses result, compared to employing a viscoelastic constitutive model in the momentum equation. The decoupled approach gathers the advantages of saving a tremendous amount of computing effort and avoiding numerical problems and flow instabilities that arise at high Weissenberg numbers, which are typical for the injection molding flow conditions. The study of such instabilities is beyond the scope of this work; see Hulsen et al. (2005).

\section{Governing equations for the injection molding problem}

The balance equations for mass, momentum, and energy are now presented and simplified with respect to the process requirements and modeling assumptions. The related constitutive equations and boundary conditions are given and justified. The general form of the balance equations for mass, momentum, and internal energy read:

$\dot{\rho}=-\rho \nabla \cdot \mathbf{u}$,

$\rho \frac{\partial \mathbf{u}}{\partial t}+\rho \mathbf{u} \cdot \nabla \mathbf{u}=\nabla \cdot \boldsymbol{\sigma}+\rho \mathbf{g}$

$\rho \dot{e}=\boldsymbol{\sigma}: \boldsymbol{D}-\nabla \cdot \mathbf{q}+\rho r+\rho h_{r} R_{c}$, where $\rho$ represents density and $\mathbf{u}$ the velocity field, $\boldsymbol{\sigma}$ the Cauchy stress tensor, $\mathbf{g}$ is the body force per unit mass, and $\dot{e}$ is the rate of change of internal energy. The terms on the right-hand side of the energy equation, Eq. 3, represent the work done to deform the material, with $\boldsymbol{D}$ the rate of deformation tensor, the heat transferred by conduction, with $\mathbf{q}$ the heat flux, the heat transferred by radiation, $r$, and internal heat generation with $R_{c}$ the reaction rate, and $h_{r}$ the reaction heat. To solve these equations, appropriate constitutive equations have to be specified for the Cauchy stress tensor, the heat flux, and an equation of state for the density and internal energy, i.e., $e=e(p, T)$, where $p$ and $T$ denote pressure and temperature, respectively, introduced in the forthcoming section. Additionally, initial and boundary conditions have to be prescribed. We now state the basic assumptions to simplify the above equations and to motivate the choice for constitutive relations.

\section{Assumptions}

The assumptions given below are quite standard for injection molding. Justification of these can be found in literature (Baaijens 1991; Douven 1991; Douven et al. 1995; Haagh and Van de Vosse 1998) and are discussed in some detail further on when appropriate.

- Compressibility effects are negligible during the filling phase.

- Flow kinematics are determined by kinematic boundary conditions.

- The melt behaves according to a generalized Newtonian flow description.

- Inertial effects are negligible.

- Thermal radiation is negligible.

- No heat source is present.

- Heat generated due to compression is negligible.

- Isotropic heat conduction.

It is known that, upon processing, the thermal conductivity of polymers is increased in the flow direction and decreased in the direction normal to the flow. Such observations were reported by, e.g., Hansen and Bernier (1972) and van den Brule and O'Brien (1990) and explained on the basis that the heat conductance is much higher along covalent bonds than throughout weak secondary bonds. Furthermore, the anisotropy resulting from the frozen-in molecular orientation can differ substantially according to the molecular structure of the polymer, being favored by linear compact polymers, and for polymers with higher relaxation times and molecular weight. All this is not taken into account due to the lack of experimental data. According to the 
above assumptions, the governing equations are simplified, yielding during the filling phase an incompressible Stokes flow problem. Thus, the continuity and momentum equation read:

$$
\begin{aligned}
& \nabla \cdot \mathbf{u}=0, \\
& \nabla p=\nabla \cdot \boldsymbol{\tau},
\end{aligned}
$$

where $\mathbf{u}$ is the velocity field and $\boldsymbol{\tau}$ is the extra stress tensor. During the packing and holding phases, the complete form of the continuity equations is solved (Eq. 1). The simplified energy equation reads:

$\rho c_{\mathrm{p}} \dot{T}=\boldsymbol{\tau}: \boldsymbol{D}+\nabla \cdot(\lambda \nabla T)$.

In injection molding, the flow kinematics are mostly determined by kinematic boundary conditions, characterized by no-slip conditions at the walls, and by a prescribed flow rate at the gate. Therefore, the precise choice of the constitutive equation for the stress tensor in the momentum equation has only a small effect on the overall kinematics as long as the shear viscosity is captured correctly. Clearly, in regions with bifurcations or close to the flow front, where significant elongation takes place, this assumption is violated. Nevertheless, a viscous approach, vs a viscoelastic approach, has the advantages of saving a tremendous amount of computing effort and avoiding numerical limitations and flow instabilities that arise at high Weisenberg numbers, which are typical for injection molding flow conditions; see Hulsen et al. (2005). The influence of viscoelastic instabilities in injection molding was notably analyzed by Grillet et al. (2002) and Bogaerds et al. (2002); however, such analyses are beyond the scope of this work. According to the generalized Newtonian flow description, the extra stress tensor $\boldsymbol{\tau}$ reads:

$\boldsymbol{\tau}=2 \eta\left(T, \boldsymbol{D}^{d}\right) \boldsymbol{D}$.

\section{Front-capturing}

In order to track the polymer/air and polymer/gas interfaces we use a front-capturing technique, also known as pseudo concentration method, which was proposed by Thompson (1986). Each material point, or infinitesimal material volume element, is labeled with a scalar $c$, and the material labels for polymer, air, and gas core are convected with the velocity $\mathbf{u}$ throughout the domain. Boundary conditions are made dependent on $c$. The method requires the addition of a pure (passive scalar) convection equation that gives the evolution of the material label distribution:

$$
\frac{\partial c}{\partial t}+\mathbf{u} \cdot \nabla c=0
$$

As initial condition, the material labels are set to zero over the entire domain $\Omega$, and at the inlet, the following boundary conditions are assigned:

$$
\begin{array}{r}
c(\mathbf{x}, t=0)=0, \quad \mathbf{x} \in \Omega, \\
c\left(\mathbf{x}, 0<t<t_{\text {fill }}\right)=1, \quad \mathbf{x} \in \Gamma_{\mathrm{e}} .
\end{array}
$$

The interface is captured for $c$ equal to 0.5 . The material properties are made dependent on the local value of the concentration, $c$, and are discontinuous across the interfaces polymer-air and polymer-gas. For the air or gas phase, $c<0.5$, the fictitious-fluid properties are assigned, while for the case $c \geq 0.5$, the polymer properties are chosen. We also perform particle tracking, using Eq. 8, but instead of prescribing at the inlet boundary a concentration value $c$, we prescribe a time label, $t$, convecting basically the flow history.

\section{Flow-induced stresses}

The total Cauchy stress tensor is the sum of an elastic and a viscous part: $\sigma=\sigma_{e}+\sigma_{v}$. The elastic part is split into a hydrostatic part and a deviatoric part: $\boldsymbol{\sigma}=-p \boldsymbol{I}+\boldsymbol{\tau}+\boldsymbol{\sigma}_{v}$. For a proper description of the polymer rheology, i.e., accurate residual stress predictions, a discrete number of relaxation times and their corresponding moduli are required; see Bird et al. (1987), Macosko (1994). Using a multi-mode description, the deviatoric part of the elastic extra stress tensor is given by:

$\boldsymbol{\tau}^{d}=\sum_{j=1}^{m} G_{j} \overline{\boldsymbol{B}}_{e j}{ }^{d}$,

in which $G_{j}$ is the relaxation modulus of a specific mode, and $\boldsymbol{B}_{e}$ the elastic Finger or conformation tensor.

Choice of the viscoelastic model: Although, in injection molding, the flow is shear-dominated, in the flow-front region, the polymer melt is stretched due to the fountain flow. The flow generated is spatially in-homogeneous, combining shear and extensional components. In literature, results reported on the calculation of flow-induced stresses in injection molding neglect fountain flow by adopting a lubrication approximation that assumes negligible velocity gradients 
parallel to the flow direction and negligible velocities across the thickness direction. However, in our model, we capture the fountain flow in the overall kinematics, and thus, we need a model that is able to capture the rheological behavior of polymers under shear and extensional flows. Likhtman and Graham (2003) presented the Rolie-Poly model for the rheology of linear polymers. Reported results show a good agreement with rheological measurements in steady and transient regimes in both shear and elongation. The model is of the differential type, and in its single-mode form, it reads:

$$
\begin{aligned}
\stackrel{\nabla}{B}_{e} & +\frac{1}{\tau_{d}}\left(\boldsymbol{B}_{e}-\boldsymbol{I}\right)+\frac{2\left(1-\sqrt{\left(3 / \operatorname{tr}\left(\boldsymbol{B}_{e}\right)\right)}\right)}{\tau_{R}} \\
& \times\left(\boldsymbol{B}_{e}+\beta\left(\frac{\operatorname{tr}\left(\boldsymbol{B}_{e}\right)}{3}\right)^{\delta}\left(\boldsymbol{B}_{e}-\boldsymbol{I}\right)\right)=\mathbf{0} .
\end{aligned}
$$

The molecular dynamic mechanisms taken into account in the derivation of this model, such as chain reptation, chain stretch, and convective constraint release, appear in the above equation, each associated with a different term, making this model particularly physically intuitive. For higher strain rates, the Rolie-Poly model produces a faster stress growth when compared to the full theory. Following the approach taken in Likhtman and Graham (2003) for a multi-mode RoliePoly description of transient experimental data, we set $\beta$ to compensate for the over prediction of the steadystate stress at large rates. In the model, two time scales are of importance: $\tau_{d}$, the disengagement (reptation) time, and $\tau_{R}$, the Rouse (stretching) time. The disengagement times are obtained from stress relaxation measurements, in which the relaxation modes are fitted. The Rouse time $\tau_{R}$ is estimated with the following relationship for mono-dispersed melts proposed by Doi and Edwards (1986):

$\tau_{R}=\frac{\tau_{d}}{3 Z}$,

in which $Z$, the number of entanglements per chain, is calculated from the weight-averaged molecular weight $M_{\mathrm{w}}$ and the averaged molar mass between entanglements $\left\langle M_{\mathrm{e}}\right\rangle$ :

$Z=\frac{M_{\mathrm{w}}}{M_{\mathrm{e}}}$

The value of $\tau_{R}$ estimated in this way is, according to Likhtman and Graham (2003), one to two times larger then those from a fit of transient experimental shear data. However, since we do not have such data, we accept our estimated value as being reasonable for our approach, where the focus is on the application to predicting residual stresses. Both choices, $\beta=0$ and $\tau_{R}$ obtained from Eq. 13, can be studied in more detail using a set of fully rheologically characterized materials. However, this is outside the scope of our paper and we restrict the injection molding analysis to the results based on these assumptions.

Since compressibility is a key issue during packing and holding phases, we next introduce a compressible version of the Rolie-Poly equation, Eq. 12. For that, we adopt the approach used by Baaijens (1991), who proposed a compressible version of the Leonov model. Assuming that the polymer cannot undergo a permanent plastic volume change, and that the deformation tensor $\boldsymbol{F}$ can be decomposed multiplicatively into an elastic part $\left(\boldsymbol{F}_{e}\right)$ and a plastic $\left(\boldsymbol{F}_{p}\right)$ part: $\boldsymbol{F}=\boldsymbol{F}_{e} \cdot \boldsymbol{F}_{p}$, the determinant of the deformation tensor $J$, is only attributed to elastic deformation:

$J_{p}=\operatorname{det}\left(\boldsymbol{F}_{p}\right)=1, \quad J_{e}=\operatorname{det}\left(\boldsymbol{F}_{e}\right)=\operatorname{det}(\boldsymbol{F})=J$.

Volumetric changes embedded in $\boldsymbol{F}_{e}$ can be split from the deviatoric response according to Simo (1987) by the following kinematic split:

$\overline{\boldsymbol{F}}_{e}=J^{-1 / 3} \boldsymbol{F}_{e}$,

in which $\overline{\boldsymbol{F}}_{e}$ is the volume preserving part of the elastic part of the deformation tensor. The elastic Finger tensor $\left(\boldsymbol{B}_{e}\right)$ relates to the elastic part of the deformations tensor $\boldsymbol{F}_{e}$ in the following manner:

$\boldsymbol{B}_{e}=\boldsymbol{F}_{e} \cdot \boldsymbol{F}_{e}^{c} ; \quad \overline{\boldsymbol{B}}_{e}=\overline{\boldsymbol{F}}_{e} \cdot \overline{\boldsymbol{F}}_{e}^{c}=J^{-2 / 3} \boldsymbol{B}_{e}$.

\section{Thermally and pressure-induced stresses}

During packing and holding phases, compressibility becomes the key phenomenon that drives the development of residual stresses. The change from filling to packing is also marked by a change in relevant mechanisms in the energy balance. Convection and viscous dissipation contributions become negligibly small, when compared with the filling phase, and heat conduction becomes the dominant thermal transport process. For the computation of thermally and pressure-induced stresses, we can simply employ a linear viscoelastic model as used by Baaijens (1991), since these originate from relatively small deformations. A viscous-elastic approach has been adopted by others; however, such models over predict stress and deformation values, since relaxation effects are neglected. In Zoetelief et al. (1996), Kamal et al. (1988), the authors have shown that a viscoelastic approach leads to a more correct position of the compressive peak and more realistic stress values in the subsurface region and at the wall. 
Only the final equations of the linear thermoviscoelastic model are presented; for a full derivation of the model, the reader is referred to Douven et al. (1995). Again, the total Cauchy stress consists of a volumetric part, which accounts for the material elastic response to changes in volume, and a deviatoric contribution, which accounts for conformation changes. It reads,

$\boldsymbol{\sigma}=-p^{h} \boldsymbol{I}+\boldsymbol{\tau}_{e}^{d}$.

When linearizing $\dot{\rho}=-\rho \operatorname{tr}(\boldsymbol{D})$, and assuming that no relevant pressure and temperature effects exist for $t \leq 0$ (before filling), an explicit relation for the hydrostatic pressure, $p^{h}$, in the solid state is found:

$p^{h}=\int_{0}^{t}\left(\frac{\alpha}{\kappa} \dot{T}-\frac{1}{\kappa} \operatorname{tr}(\boldsymbol{D})\right) d t$,

where $\alpha$ is the thermal expansion coefficient and $\kappa$ the isothermal compressibility coefficient, which are defined as:

$\alpha=-\frac{1}{\rho}\left(\frac{\partial \rho}{\partial T}\right)_{p}$,

$\kappa=\frac{1}{\rho}\left(\frac{\partial \rho}{\partial p}\right)_{T}$,

respectively.

The temperature dependence of the material's viscoelastic behavior and is described using the timetemperature superposition, i.e., assuming it to be thermorheologically simple, implying that the relaxation times and viscosities are shifted from a reference temperature $T_{0}$ to the current temperature $T$, by a shift factor $a_{T}$ :

$\eta_{j}=a_{T}(T) \eta_{j 0}, \quad \tau_{j}=a_{T}(T) \tau_{j 0}$,

with $\eta_{j 0}$ and $\tau_{j 0}$ denoting the viscosity and relaxation time at the reference temperature, $T_{0}$. Only for $T \geq T_{\mathrm{g}}$ is the shift factor determined by the WLF equation; for
$T<T_{\mathrm{g}}$, the relaxation times become so large that we set $T_{\mathrm{g}}$ as the lower bound limit for the time temperature superposition. Hence, for $T<T_{\mathrm{g}}, a_{T}=a_{T}\left(T_{\mathrm{g}}\right)$. The WLF equation is given by:

$\log a_{T}(T)=\frac{-C_{1}\left(T-T_{0}\right)}{C_{2}+T-T_{0}}$.

When no deformation effects before $t=0$ exist and assuming the material is thermorheologically simple, the deviatoric part $\boldsymbol{\tau}^{d}$ of the Cauchy stress tensor can be written as:

$\boldsymbol{\tau}^{d}=\sum_{j=1}^{m} \boldsymbol{\tau}_{j}^{d}, \boldsymbol{\sigma}_{j}^{d}=2 \int_{0}^{t} G_{j 0} e^{-\left(\xi_{i j}-\xi_{s j}\right)} \boldsymbol{D}^{d}(s) d s, G_{j 0}=\frac{\eta_{j 0}}{\tau_{j 0}}$,

where $\xi_{q j}$ is the reduced relaxation modulus, which, according to the linear Maxwell model, is defined as:

$\xi_{q j}=\int_{o}^{q} \frac{1}{a_{T}(s) \tau_{j}} d s, \quad q=t, s$.

The thermo-linear viscoelastic model is only solved for $T \leq T_{\mathrm{g}}$. Above the glass transition temperature, the residual stresses are isotropic and equal to minus the pressure in the melt. The computation of thermally and pressure-induced stresses can be substantially simplified by considering a series of assumptions commonly employed:

1. The material is assumed to stick to the mold for as long the pressure in the symmetry line remains positive.

2. Continuity of stress and strain at the solid melt interface.

3. The normal stress $\sigma_{22}$, see Fig. 1, is constant across the part thickness and equals minus the pressure in

Fig. 1 Sketch of the cavity geometry

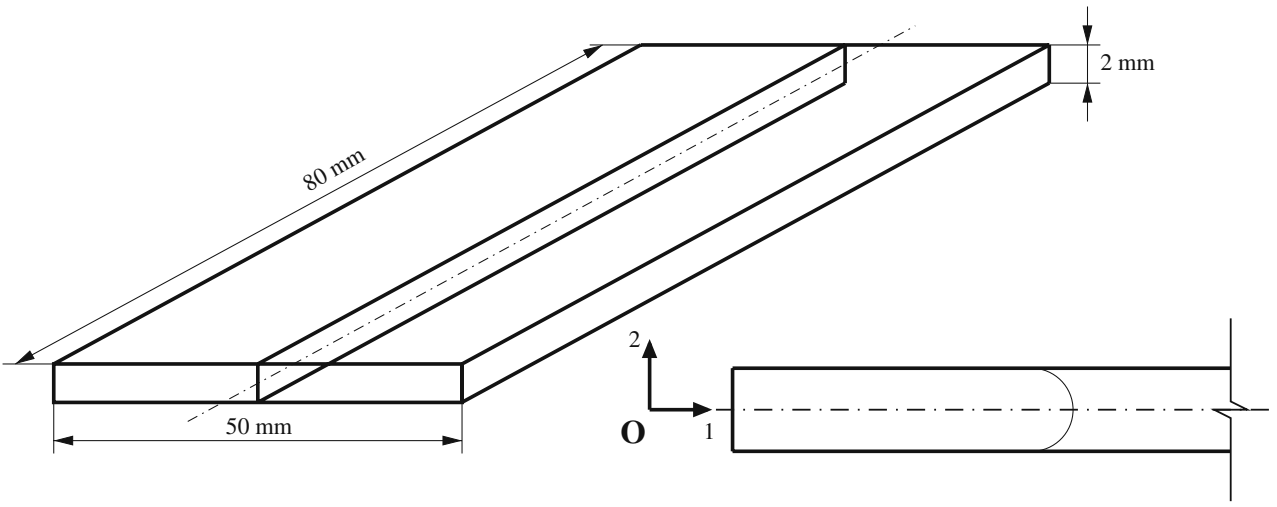


the melt as long as the temperature at the symmetry line in the mold, $T^{*}$, is larger than the glass transition temperature $T_{\mathrm{g}}$.

4. In a coordinate system with the 22 direction perpendicular to the filling direction ${ }_{11}$, the shear strain component $\varepsilon_{12}$ is disregarded.

5. As long as pressure remains above zero, at the symmetry line, the only non-zero strain component is $\varepsilon_{22}$.

6. Solidification takes place when the no-flow temperature $\left(T_{g}\right)$ is reached.

7. Mold elasticity is disregarded.

8. Frozen-in or flow-induced stresses can be neglected.

A detailed discussion on these assumptions is given by Baaijens (1991); we do not want to repeat all this in this paper except for the last two assumptions. By disregarding the mold, elasticity errors are introduced in the pressure history inside the mold cavity. This will, of course, have an effect on the development of the pressure-induced stresses. Baaijens (1991) showed that introducing mold elasticity slows the decay of the pressure inside the mold cavity, causing some changes in the final profile of the thermally and pressureinduced stresses. Regarding assumption 8 , it was shown in Baaijens (1991) and Zoetelief et al. (1996) that the order of magnitude of flow-induced stresses is about $10^{2}$ lower than the thermally and pressure-induced stresses. Moreover, the addition of flow-induced stresses to the total residual stresses is still a matter of some debate. Zoetelief et al. (1996) used the removal layer technique to measure residual stresses parallel and perpendicular to flow direction. They found a difference of less than $20 \%$, suggesting that the influence of flow-induced stresses is small.

\section{Injection molding case}

To evaluate the development of flow-induced stresses in GAIM parts, we depart from an earlier study, Baaijens (1991), in which residual stresses were computed for injection-molded PC plates. Baaijens used a Hele-Shaw formulation to predict the flow kinematics and used the Leonov model to compute viscoelastic flow-induced stresses. In our study, we apply a fully 3-D model to the same injection problem, i.e., geometry, material, and processing conditions, to study the development of flow-induced stresses when using conventional or GAIM. Small differences are obviously expected when using a fully 3-D-based approach without the Hele-Shaw assumptions, which are: constant pressure across the mold thickness, negligible velocity in the thickness direction, small velocity gradients, and negligible thermal conduction parallel to the mid plane. The main difference, however, is that we capture the fountain flow, and the melt stretched at the flow front region will contribute to the final flow-induced stress profiles. To compare with the results of Baaijens, we have only to investigate a 2-D problem, of a cross section of the original 3-D geometry of $80 \times 2 \mathrm{~mm}$ (length, height); see Fig. 1. The gate is located at the channel entrance. In Baaijens (1991), flow-induced stresses were computed in an injection-molded PC plate. Next, we study the effect of GAIM on flow-induced stresses, using the same material, processing conditions, and geometry. Again, we approximate the original 3-D geometry to a 2-D problem by taking a cross-section along the channel's length; see Fig. 1. The gate occupies the total height of the channel.

\section{Constitutive relations}

Viscoelastic model: The properties of the PC grade, taken from Baaijens (1991), are given in Table 1. To estimate the Rouse time, additional information is needed, namely, the weight-averaged molecular weight $M_{\mathrm{w}}$ and the averaged molar mass between entanglements $\left\langle M_{\mathrm{e}}\right\rangle$ of PC. Since, in Baaijens (1991), there is no specification of the material grade used, we took the

Table 1 Material parameters for PC

\begin{tabular}{|c|c|c|c|}
\hline \multicolumn{4}{|c|}{ Parameters WLF equation: } \\
\hline$T_{0}=200$ & \multicolumn{3}{|l|}{$\left[{ }^{\circ} \mathrm{C}\right]$} \\
\hline$C_{1}=-4.217$ & \multicolumn{3}{|l|}{$[-]$} \\
\hline$C_{2}=94.95$ & \multicolumn{3}{|l|}{$\left[{ }^{\circ} \mathrm{C}\right]$} \\
\hline \multicolumn{4}{|l|}{ Thermal properties: } \\
\hline$c_{\mathrm{p}}=1500$ & \multicolumn{3}{|l|}{$\left[\mathrm{J} \mathrm{kg}^{-1}\right]$} \\
\hline$\lambda=0.27 \cdot 10^{-3}$ & \multicolumn{3}{|c|}{$\left[\mathrm{J} \mathrm{s}^{-1} \mathrm{~mm}^{-1} \mathrm{~K}^{-1}\right]$} \\
\hline \multicolumn{4}{|c|}{ Viscoelastic properties: } \\
\hline$\eta^{\prime}=3000 \bar{\eta}$ & {$[\mathrm{Pas}]$} & & \\
\hline$\tau_{1}=10^{-1}$ & {$[\mathrm{~s}]$} & $\eta_{1}=9.7410^{3}$ & {$[\mathrm{Pas}]$} \\
\hline$\tau_{2}=10^{-2}$ & {$[\mathrm{~s}]$} & $\eta_{2}=6.7510^{3}$ & {$[\mathrm{Pas}]$} \\
\hline$\tau_{3}=10^{-3}$ & {$[\mathrm{~s}]$} & $\eta_{3}=1.2510^{3}$ & {$[\mathrm{Pas}]$} \\
\hline$M_{\mathrm{w}}=28.5$ & {$\left[\mathrm{~kg} \mathrm{~mol}^{-1}\right]$} & & \\
\hline$M_{\mathrm{e}}=1.790$ & {$\left[\mathrm{~g} \mathrm{~mol}^{-1}\right]$} & & \\
\hline$\tau_{\mathrm{R}}=2.22 \cdot 10^{-3}$ & {$[\mathrm{~s}]$} & & \\
\hline \multicolumn{4}{|l|}{ Tait parameters: } \\
\hline$s=0.51 \cdot 10^{6}$ & {$\left[{ }^{\circ} \mathrm{CPa}^{-1}\right]$} & & \\
\hline solid & melt & & \\
\hline$a_{o}=868 \cdot 10^{-6}$ & $86810^{-6}$ & {$\left[\mathrm{~m}^{3} \mathrm{~kg}^{-1}\right]$} & \\
\hline$a_{1}=0.22 \cdot 10^{-6}$ & $0.57710^{-6}$ & {$\left[\mathrm{~m}^{3} \mathrm{~kg}^{-1} \mathrm{~K}^{-1}\right]$} & \\
\hline$B_{o}=395.4 \cdot 10^{6}$ & $316.110^{6}$ & {$[\mathrm{~Pa}]$} & \\
\hline$B_{1}=2.609 \cdot 10^{-3}$ & $4.07810^{-3}$ & {$\left[{ }^{\circ} \mathrm{C}^{-1}\right]$} & \\
\hline
\end{tabular}


value of $M_{\mathrm{w}}$ and $M_{\mathrm{e}}$ from literature. In Souheng $\mathrm{Wu}$ (1989), the averaged molar mass between entanglements $\left\langle M_{\mathrm{e}}\right\rangle$ of PC was determined, and a value equal to $1.790 \mathrm{~kg} \mathrm{~mol}^{-1}$ was found. In Klompen (2005), the $M_{\mathrm{w}}$ of a similar injection molding PC grade is given, and equals $25.8 \mathrm{~kg} \mathrm{~mol}^{-1}$. Inserting these values in Eqs. 13 and 14, a value for the Rouse time $\tau_{R}$ equal to $2.22 \cdot 10^{-3} \mathrm{~s}$ is found for the highest relaxation mode. For the other relaxation modes, the values of the corresponding stretching relaxation times are found to be too small to be relevant for the strain rates involved in the process. Thus, with the exception of the highest relaxation mode, stretching can be neglected and a nonstretch form of the Rolie-Poly equation can be adopted instead:

$$
\stackrel{\nabla}{\overline{\boldsymbol{B}}_{e}}+\frac{1}{\tau_{d}}\left(\overline{\boldsymbol{B}}_{e}-\boldsymbol{I}\right)+\frac{2}{3} \operatorname{tr}\left(\boldsymbol{L} \cdot \overline{\boldsymbol{B}}_{e}\right)\left(\overline{\boldsymbol{B}}_{e}+\beta\left(\overline{\boldsymbol{B}}_{e}-\boldsymbol{I}\right)\right)=\mathbf{0} .
$$

Specific volume: To describe changes in specific volume, we use the so-called Tait model, see Zoller (1982), which has been used vastly for amorphous polymers. The model equation reads:

$v(p, T)=\left(a_{0}+a_{1}\left(T-T_{\mathrm{g}}\right)\right) \times\left(1-0.0894 \ln \left(1+\frac{p}{B}\right)\right)$,

in which $T_{\mathrm{g}}$ is the glass transition temperature, given by $T_{\mathrm{g}}(p)=T_{\mathrm{g}}(0)+s p$, and the parameter $B(T)$ by $B(T)=B_{0} \exp \left(-B_{1} T\right)$. The equation parameters $a_{0}, a_{1}$, $B_{0}$, and $B_{1}$ are different for the melt $\left(T>T_{\mathrm{g}}\right)$ and the solid state $\left(T<T_{\mathrm{g}}\right)$. The total set of parameters is fitted on PVT experiments that are run at quasi equilibrium conditions, under which $v$ is measured at varying pressure and temperature. History effects are therefore not taken into account. For a detailed explanation on the model parameters, the reader is referred to Zoller (1982).

Viscosity model: The generalized Newtonian viscosity, Eq. 7, is computed from the steady Leonov model at simple shear Baaijens (1991),

$\tilde{\eta}(\dot{\gamma}, T)=\eta_{0} a_{T}+\sum_{k=1}^{n} \frac{2 \eta_{k} a_{T}}{1+x_{k}}$,

with $x_{k}$ defined as:

$x_{k}=\sqrt{1+4\left(\theta_{k} a_{T} \dot{\gamma}\right)^{2}}$.

The relaxation times $\theta_{j}$ are shifted by $a_{T}$, which is computed by the WLF equation, Eq. 23 .
Boundary conditions

Assuming a computational domain $\Omega$, Fig. 2, boundary conditions are specified at $\Gamma_{\mathrm{e}}, \Gamma_{\mathrm{w}}$, and $\Gamma_{\mathrm{v}}$, designating the mold entrance, mold walls, and the air vents, respectively. We prescribe a volume flow rate while filling the mold cavity, by means of a fully developed velocity profile, and an imposed pressure (i.e., normal stress) during the packing and holding phases. At the mold walls, we use adjustable Robin boundary conditions that allow the change from slip to no-slip depending on the material label $c$ at the wall. If air touches the wall, $c=0$, a slip boundary condition is assigned. For polymer, $c \geq 0.5$, a no-slip condition is imposed by setting a traction force at the wall. Accordingly, the boundary condition for the velocity and stress components $u_{\mathrm{t}}$ and $\sigma_{\mathrm{t}}$ in tangential direction read:

$a u_{\mathrm{t}}+\sigma_{\mathrm{t}}=0 \quad \forall \mathbf{x} \in\left(\Gamma_{\mathrm{w}} \cup \Gamma_{\mathrm{v}}\right)$,

in which the dimensionless "Robin penalty parameter" $a$ is defined as

$a=a(c)= \begin{cases}\geq 10^{6} & \text { if } c \geq 0.5: \text { no slip or leakage } \\ 0 & \text { if } c<0.5: \text { slip or leakage }\end{cases}$

Air is only allowed to exit the cavity at air vents, $\Gamma_{\mathrm{v}}$. For this, a Robin condition is assigned for the velocity and stress components $u_{\mathrm{n}}$ and $\sigma_{\mathrm{n}}$ in normal direction:

$$
\begin{gathered}
u_{\mathrm{n}}=0 \quad \forall \mathbf{x} \in \Gamma_{\mathrm{w}} \\
a u_{\mathrm{n}}+\sigma_{\mathrm{n}}=0 \quad \forall \mathbf{x} \in\left(\Gamma_{\mathrm{w}} \cup \Gamma_{\mathrm{v}}\right),
\end{gathered}
$$

in which $a$ is again given by Eq. 31. However, in this case, the term "slip" should be replace by "leakage."

An initial temperature field is prescribed over the entire domain corresponding to the air/fictitious fluid phase,

$T_{i}=T_{0}(\mathbf{x}, t=0) \quad \mathbf{x} \in \Omega$.

At the injection gate $\Gamma_{\mathrm{e}}$, the injection temperature is prescribed,

$T=T_{\mathrm{e}}(\mathbf{x}, t) \quad \in \Gamma_{\mathrm{e}}, t>0$.

$\Gamma_{\mathrm{w}}$

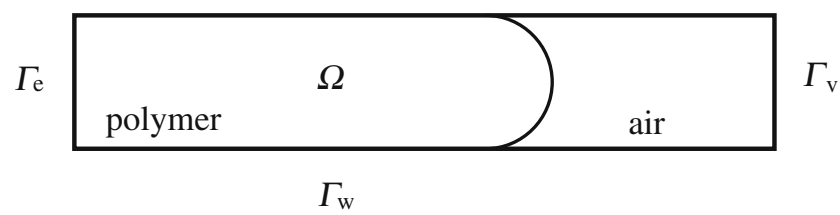

Fig. 2 Computational domain 
At the mold walls, we prescribe a Dirichlet boundary, via which a constant temperature is assigned,

$T=T_{\mathrm{w}}(\mathbf{x}, t=0, t) \quad \mathbf{x} \in \Gamma_{\mathrm{w}} \cup \Gamma_{\mathrm{v}}, t \geq 0$.

As a boundary condition for the computation of flowinduced stresses, we assume that the material injected inside the mold cavity carries no deformation history. This assumption is not consistent with the fully developed velocity profile prescribed at the inlet. However, given the high temperatures in the melt prior to injection, the assumption that any deformation history in the material is erased is a reasonable one. Thus, the boundary condition for the stress problem reads:

$\overline{\boldsymbol{B}}_{e}=\boldsymbol{I} \quad \forall \mathbf{x} \in \Gamma_{\mathrm{e}}, t \geq 0$.

Thermally and pressure-induced stresses:

post-ejection structural analysis

In our study, we only focus on the development of residual stresses inside the mold while the molded part is still kept under pressure. The situation when, due to the absence of pressure, the part is allowed to move and consequently shrink inside the mold is disregarded. The ejection phase, in which the part is ejected from the mold cavity and allowed to shrink and deform accordingly to the residual stress field resulting in warpage, is dealt in a separate structural analysis. We follow the so-called residual-stress methods, in which computed residual stresses are used as loading conditions in a structural analysis, to find the equilibrated residual stress profile and corresponding displacement field. In a different approach, Baaijens (1991) and Douven et al. (1995) computed displacements using shell type of elements. More recently, Kennedy (2008) proposed a hybrid model in which shrinkage data measured on injection-molded specimens are used to calibrate computed residual stresses.

Boundary conditions employed inside the mold, during filling, packing, and holding phases, and those after the ejection phase employed in the structural analysis (addressed in a separate section) have to be considered. For the injection phases, filling, packing, and holding, two situations are distinguished for which adequate boundary conditions have to be prescribed:

- Constrained quench with molten core-The pressure inside the cavity is positive and the temperature in the nodes located at the symmetry line of the part, $T^{*}$, is still above the glass transition temperature $T_{\mathrm{g}}$.

$p \geq 0, \quad T^{*} \geq T_{\mathrm{g}}(p)$.
All strain components are zero except for $\varepsilon_{22}$, which must obey $\int_{h / 2}^{h / 2} \varepsilon_{22} d x_{2}=0$. With respect to the local coordinate system, the Cauchy stress components read:

$\sigma_{22}=-p, \quad \sigma_{11}=\tilde{\sigma_{11}}+\frac{b}{a}\left(-p-\tilde{\sigma_{22}}\right)$.

- Constrained quench with solid core-The pressure inside the cavity is positive but the temperature in the nodes located at the symmetry line of the part, $T^{*}$, is below the glass transition temperature $T_{\mathrm{g}}$ :

$p \geq 0, \quad T^{*}<T_{\mathrm{g}}(p)$.

The material still contacts the wall; the only difference with the previous situation is that $\sigma_{22}$ is prescribed according to Eq. 40, assuming $\int_{h / 2}^{h / 2} \varepsilon_{22} d x_{2}=$ 0 . The Cauchy stress components read:

$$
\begin{gathered}
\sigma_{22}=\frac{\int_{h / 2}^{h / 2} \tilde{\sigma_{22} / a d x_{2}}}{\int_{h / 2}^{h / 2} 1 / a d x_{2}} . \\
\sigma_{11}=\tilde{\sigma_{11}}+\frac{b}{a}\left(\sigma_{22}-\tilde{\sigma_{22}}\right) .
\end{gathered}
$$

In order to find the equilibrated residual stress profile after ejection, we perform a geometrically nonlinear analysis using the commercial finite element package MSC Marc. Residual stress values obtained during the filling, packing, and holding phases are interpolated to Gaussian points, imposing on the part a non-equilibrium stress state. The domain is spatially discretized with four-noded bilinear quadrilateral elements, and the material behavior is described by a linear-elastic constitutive equation. The Poisson ratio for PC $v$ is taken to be 0.37; see van Krevelen (1990). For the gas-assisted injection case, since after the gas injection no polymer is injected to seal the gate, we cannot simply run a structural analysis on the entire

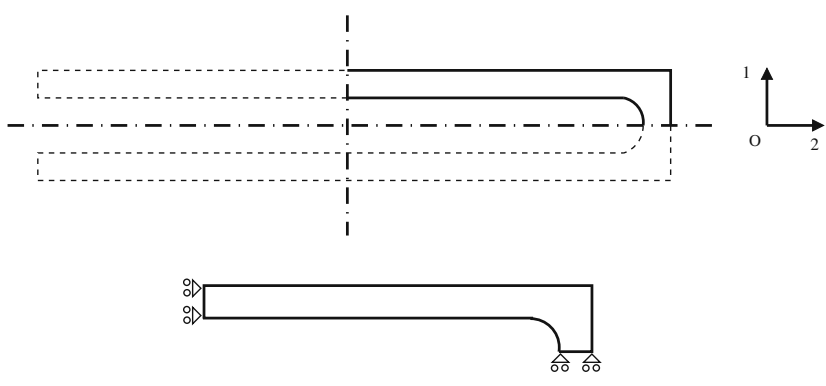

Fig. 3 Boundary conditions employed in the non-linear geometrical analysis for the GAIM case 


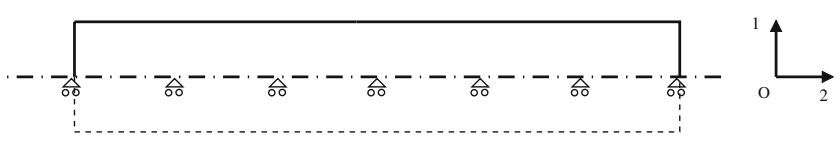

Fig. 4 Boundary conditions employed in the non-linear geometrical analysis for the conventional injection case

domain. However, given the homogeneity of the stress field throughout the part's length, see Fig. 15, we can assume it to be symmetric over the length. In Fig. 3, we illustrate the computational domain with the proper boundary conditions to exclude rigid body motions. For the conventional injection case, no symmetry assumption can be used over the length, since the residual stress profile changes throughout the part's length. We therefore carry out a dynamic analysis, in which constraints of rigid body motions are not required. Since the acceleration values are very small, inertia forces negligibly affect the computed stress field. The part is mechanically supported, as shown in Fig. 4. As output of the analysis, Cauchy stresses and logarithmic strains are obtained. The magnitude of the computed strains is of $\mathscr{O} \sim 10^{-3}$, thus validating the use of a linearelastic approach. A viscoelastic approach would have been more consistent within the modeling framework presented here. However, it was the scope of this study to analyze the development of residual stresses inside the mold during the filling, packing, and holding injection phases. The structural analysis carried out upon ejection is only done so that an equilibrium stress state is obtained.

\section{Processing conditions}

\section{Conventional injection molding}

The polymer is injected at an average velocity of $120 \mathrm{~mm} \mathrm{~s}^{-1}$ at $320^{\circ} \mathrm{C}$. After filling, $t_{\mathrm{f}}=0.67 \mathrm{~s}$, a short packing phase, $t_{\mathrm{p}}=0.71 \mathrm{~s}$, follows until an injection pressure of $50 \mathrm{MPa}$ is reached. This pressure is then maintained during a holding phase of $4 \mathrm{~s}$. After this time, the gate is assumed to freeze off instantaneously. The temperature at the mold walls is set to $80^{\circ} \mathrm{C}$. In Table 2, the processing conditions are summarized.

Table 2 Characteristic values of injection molding process variables for thermoplastics

\begin{tabular}{|c|c|c|c|c|}
\hline \multicolumn{2}{|l|}{$\begin{array}{l}\text { Packing pressure } \\
{[\mathrm{MPa}]}\end{array}$} & \multirow[t]{2}{*}{$\begin{array}{l}\text { Holding time } \\
{[\mathrm{s}]}\end{array}$} & \multicolumn{2}{|c|}{$\begin{array}{l}\text { Avg velocity } \\
{\left[\mathrm{mm} \mathrm{s}^{-1}\right]}\end{array}$} \\
\hline & & & Polymer & Gas \\
\hline Conventional & 50.0 & 4 & 120 & - \\
\hline GAIM & 0.145 & 2 & 120 & 130 \\
\hline
\end{tabular}

\section{Gas-assisted injection molding}

The conditions used for GAIM, in terms of injection temperature and speed, are equal to those used in the conventional case apart from the injection time that is set to $0.44 \mathrm{~s}$. The gas is injected over a limited height of the inlet $(1 \mathrm{~mm})$ and its injection speed is set to a value slightly higher than that of the polymer. The gas is injected immediately after the polymer injection; thus, there is no delay time between the polymer and the gas injections. The remaining processing conditions are listed in Table 2.

\section{Computational aspects}

We use a finite element solution algorithm to solve the flow and heat transfer problems in 3-D, developed earlier in our group by Haagh and Van de Vosse (1998). The Stokes and energy equation are coupled but solved within each time step in a staggered manner. The Stokes equations, Eqs. 1 and 5, that compose the flow problem are solved by a velocity-pressure formulation that is discretized by a standard Galerkin finite element method (GFEM). Since, during the filling phase, the flow is incompressible, and in the subsequent phases (packing and holding) compressible, two different weak forms are found after performing the Galerkin finite element discretization. The system of equations is solved in an integrated manner; both velocity and pressure are treated as unknowns. In case of 2-D computations, the discretized set of algebraic equations is solved using a direct method based on a sparse multi-frontal variant of Gaussian elimination (HSL/MA41) - direct solver (HSL); for details, the reader is referred to Amestoy and Duff (1989a, b) and Amestoy and Puglisi (2002). In 3-D computations, the resulting system of linear equations consists of generally large sparse matrices, and often, iterative solvers are employed, which use successive approximations to obtain a convergent solution. Furthermore, they avoid excessive CPU time and memory usage. In our 3-D computations, we use a generalized minimal residual solver (GMRES), see Saad and Schultz (1992), in conjunction with an incomplete LU decomposition preconditioner. The computational domain is discretized with elements with discontinuous pressure of the type Crouzeix-Raviart- $Q_{2} P_{1}^{d}$, 2-D quadrilateral or brick 3-D finite elements, in which the velocity is approximated by a continuous piecewise polynomial of the second degree, and the pressure by a discontinuous complete piecewise polynomial of the first degree. The degrees of freedom at the nodal points correspond to the velocity components, 
while, at the central node, the pressure and pressure gradients are computed. The integration on the element is performed using a nine-point (2-D) or 27-point (3-D) Gauss rule. Special care has to be given to solve the front-capturing convection equation. Convectiondominated problems give rise to unstable solutions with spurious node-to-node oscillations, referred to as wiggles. To overcome this problem, the streamlineupwind Petrov-Galerkin (SUPG) method, proposed by Brooks and Huges (1982), is the most employed and, thus, is adopted in our model. Flow-induced stresses are computed by adopting an explicit scheme, which is presented in Appendix A, to numerically integrate the Rolie-Poly equation. For the numerical solution of thermally and pressure-induced stress problem, the linear thermo-viscoelastic constitutive model is written in an incremental form; see Baaijens (1991); Douven et al. (1995). In this way, the stress state at time $t_{n+1}$ can be evaluated using the completely determined stress state at $t_{n}$, together with the pressure and temperature history at $t_{n+1}$. Details of the incremental form are given in Appendix B.

\section{Results \& discussion}

Flow-induced residual stresses

\section{Conventional injection molding}

In Fig. 5, we show the first normal stress difference N1, defined as N1 $=\boldsymbol{\tau}_{11}-\boldsymbol{\tau}_{22}$, computed at the end of the filling phase. The results obtained by Baaijens (1991) are also shown. The most important difference is the high value of N1 close to the mold wall, which results from the contribution of the fountain flow. Baaijens (1991) used a Hele-Shaw-based injection model that only takes into account shear contributions, and consequently, the $\mathrm{N} 1$ profile evidences a single peak halfway in between the part mid-plane and the mold wall, where shear rates attain their maximum. Such a profile is not in agreement with experimental observations, see Kamal and Tan (1979), in terms of measured flowinduced birefringence, since it fails to predict the highly oriented skin layer, which is induced by the steady elongational flow in the advancing flow front; see, e.g., Tadmor (1974). The material that is deposited at the skin layers travels through the center region of the flow, where shear rates are minimal, before it is stretched at the flow front and subsequently quenched at the walls. Our model fully captures the spatial inhomogeneity of the flow field, and thus, both shear and extensional components contribute to the final $\mathrm{N} 1$ profile. The influence of the fountain flow on the flow kinematics is illustrated in Fig. 6, by plotting time labels during the polymer injection. Figure 7 shows the first normal stress difference profiles at increasing upstream distances from the flow front. The effect of fountain flow on flow-induced stresses is depicted by a negative N1 value in the center line, resulting from the compression the material undergoes as it approaches the flow front, and large and positive N1 values close to the wall, denoting the extension of the fluid elements that are

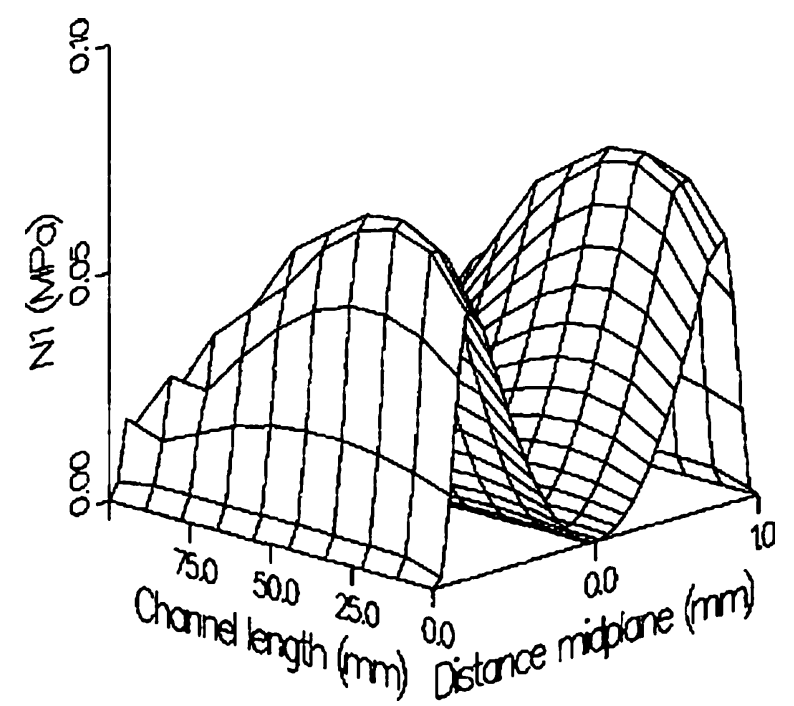

(a)

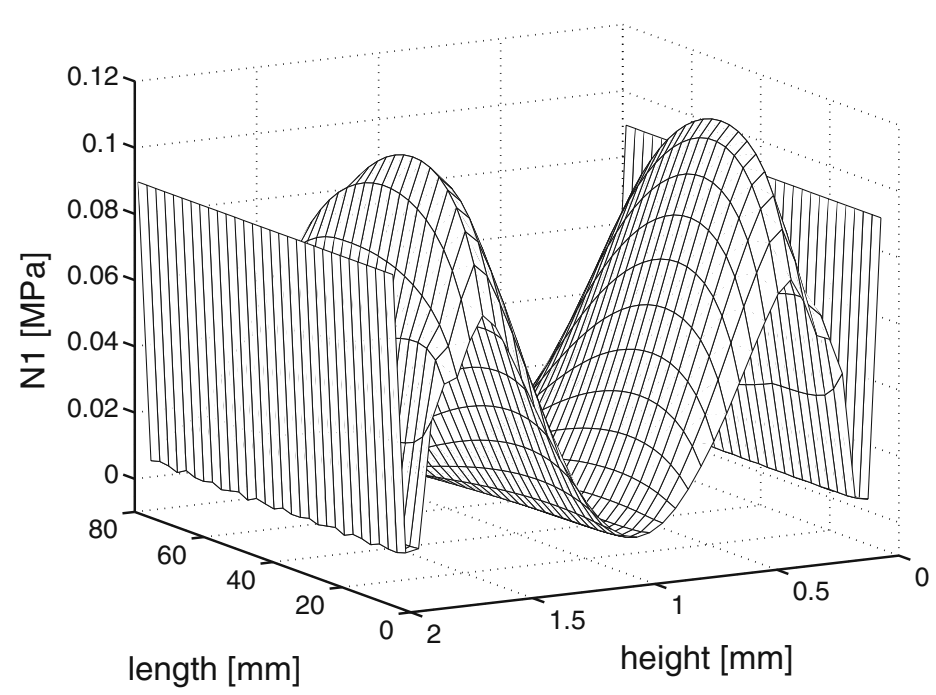

(b)

Fig. 5 First normal stress difference (N1) results at the end of the filling phase: a results computed by Baaijens (1991) and b from our simulation 


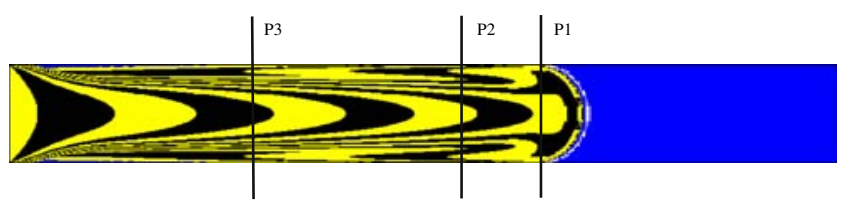

Fig. 6 Time labels during injection, denoting the contribution of fountain flow to the flow kinematics

deposited in the skin layer. In regions where there is no influence of the fountain flow, here designated as fully developed flow regions, N1 values are zero at the center line. The large stress values at the walls result also from the contribution of the singularity at the contact point between the polymer interface and the wall. The effect of this singularity on the stress values increases upon mesh refinement, resulting in unrealistically large stress values at the walls. Similar observations were reported by Mavridis et al. (1988). A way to exclude the effect of the contact-point singularity from the overall stress computations would be to set $\overline{\boldsymbol{B}}_{e}=\boldsymbol{I} \forall c<1$, implying that stresses could only develop behind the flow front. However, such an approach would exclude the local phenomena occurring at the flow front, $0.5<$ $c<1.0$, and lead to zero stress values at the walls, which are not meaningful. The stress values at the walls reported here are thus only indicative, but since they qualitatively agree with the experimentally observed trends, we include them in our results.

Regarding the shear-induced peaks in Fig. 5, it can be seen that we predict higher values than Baaijens' results, although the magnitude of the stresses is similar. The fact that we solve the 3-D problem without using

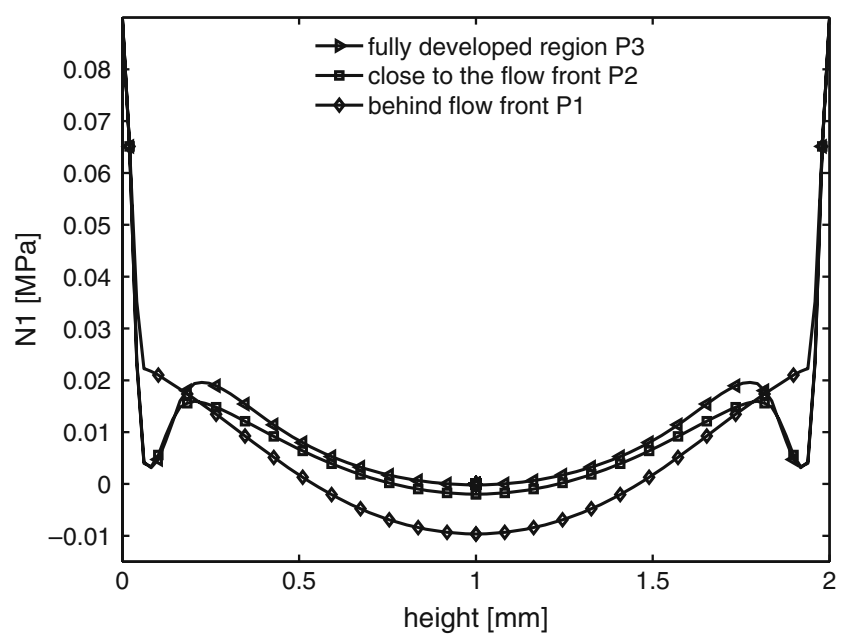

Fig. $7 \mathrm{~N} 1$ profiles across the channel height in the vicinity of the flow front and at increasing upstream distances from the flow front, positions 1, 2, and 3 according to Fig. 6

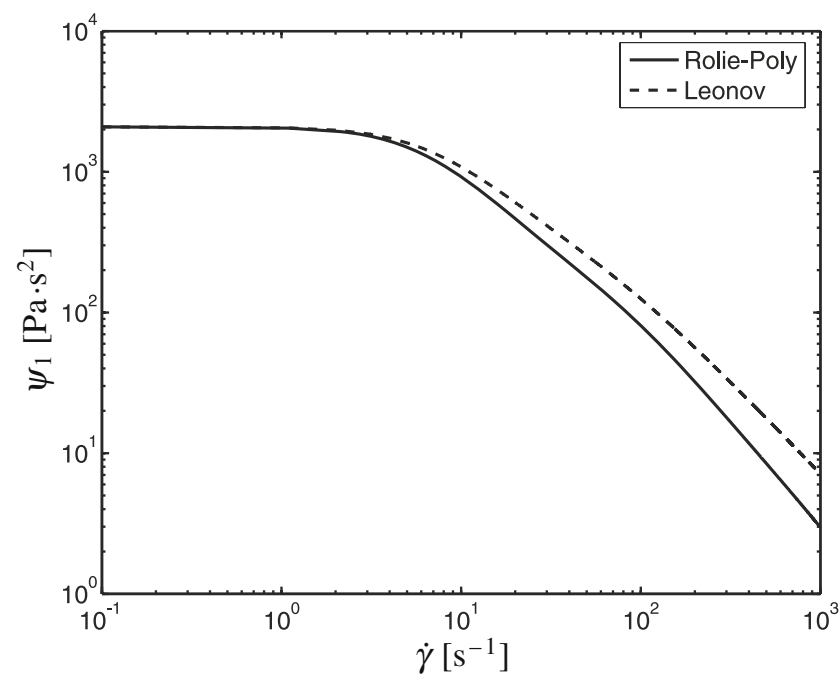

Fig. 8 First normal stress coefficient $\psi_{1}$ predicted by the Leonov and Rolie-Poly model

the Hele-Shaw assumptions will, to a certain degree, contribute to this effect. Though the Hele-Shaw model accurately predicts pressure fields for thin-walled parts, as is the case of the simulation here, the predicted temperature field can be erroneous, due to the absence of the fountain flow, causing the heat convected from the core to the walls in the flow front to not be taken into account. In the case of our simulations, this could cause a faster decrease in the cross-sectional height, caused by a thicker solidified layer, which might be responsible for higher shear rates, therefore inducing higher values of N1. Additionally, one might expect the choice of the viscoelastic model to influence the stress results since we use a compressible version of the Rolie-

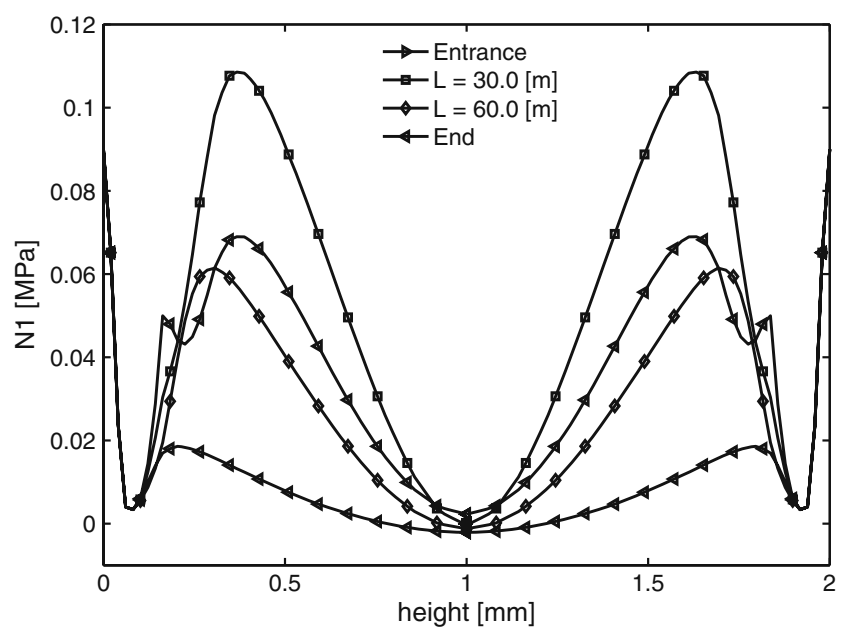

Fig. 9 N1 profiles across the channel height for different positions along the cavity length 


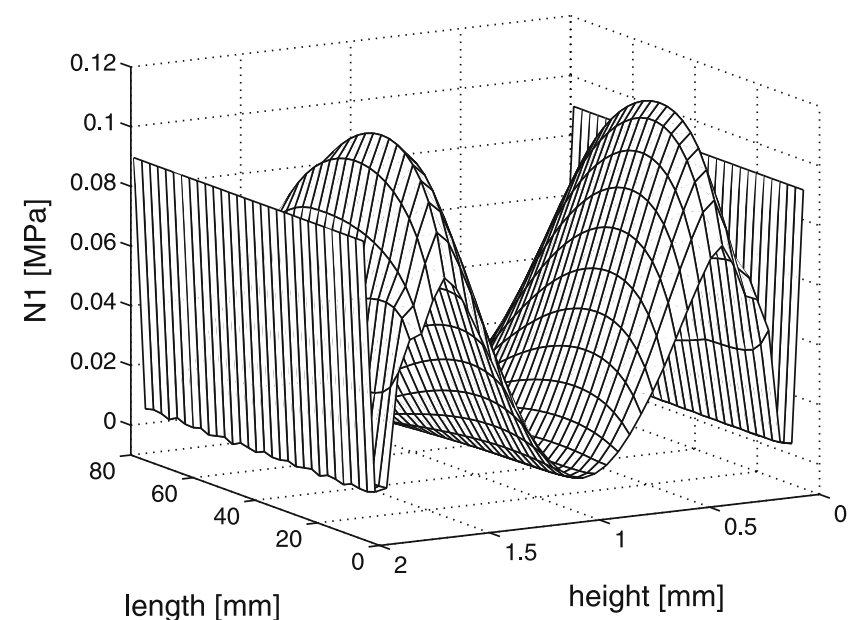

(end of filling)

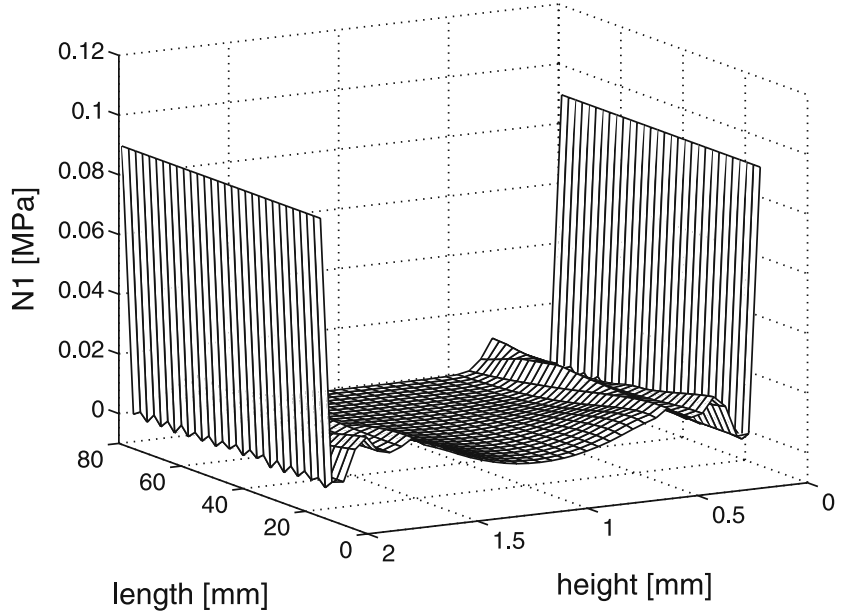

$t=2.0 \mathrm{~s}$

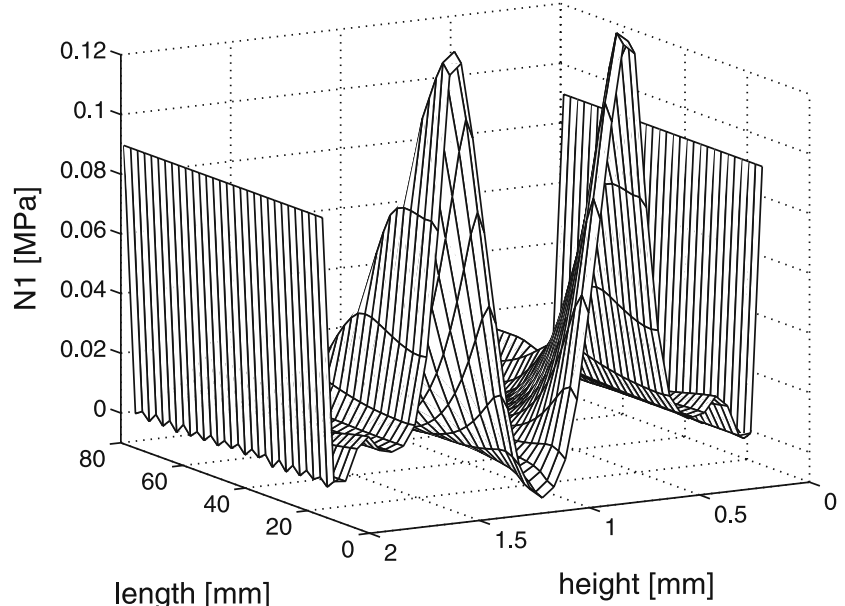

$t=3.0 \mathrm{~s}$

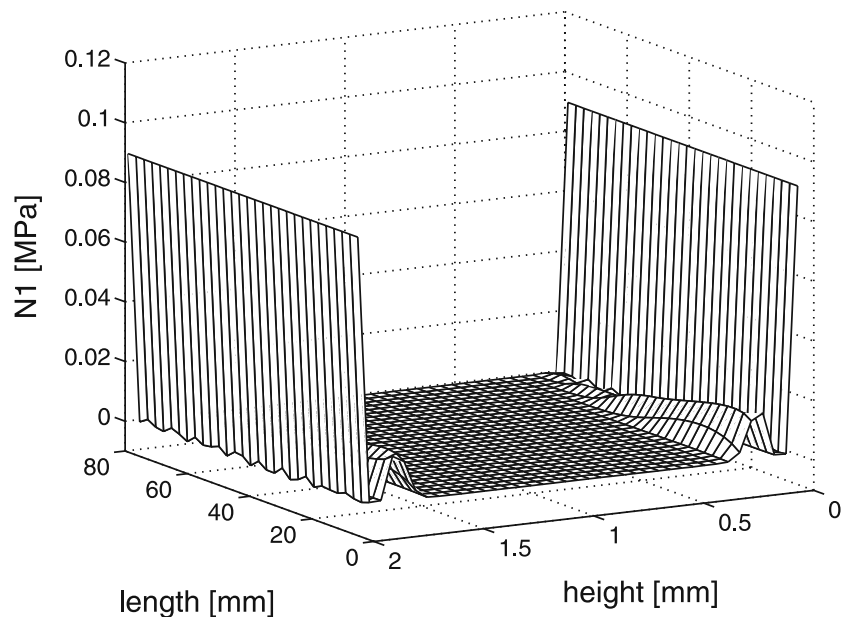

(end of packing)

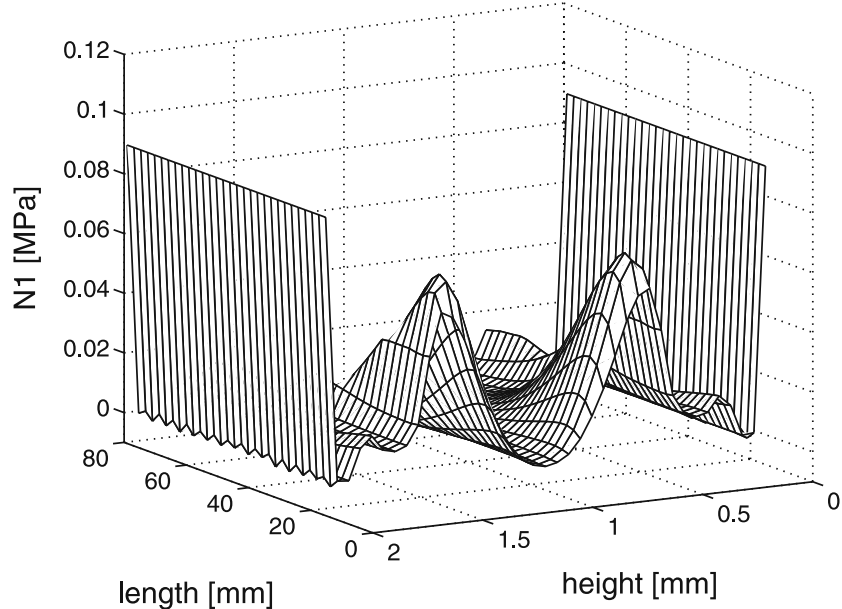

$t=2.5 \mathrm{~s}$

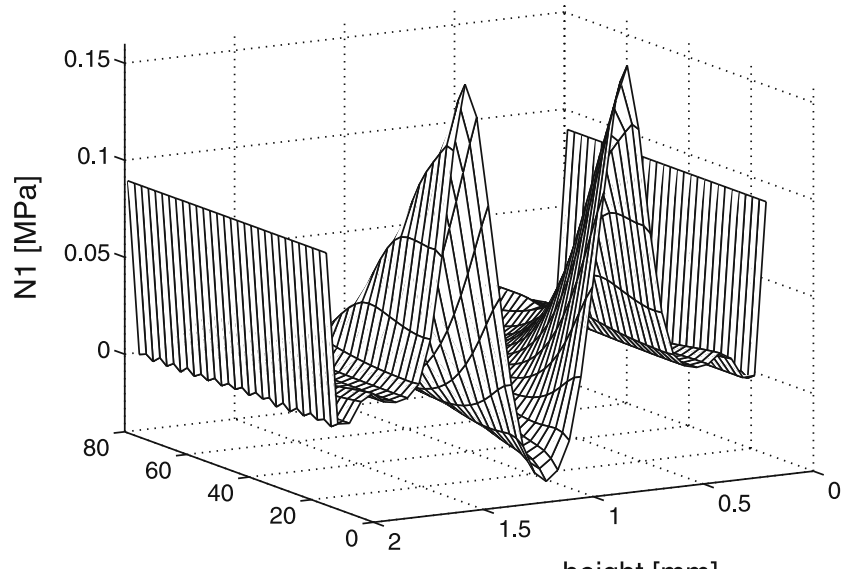

length [mm]

$$
t=4.0 \mathrm{~s}
$$

Fig. 10 Time evolution of N1 in the convention injection molding case 
Poly model instead of the compressible Leonov model employed in the study of Baaijens (1991). To investigate the influence of the viscoelastic model, we plot in Fig. 8 the first normal stress coefficient, defined as $\psi_{1}=\mathrm{N} 1 / \dot{\gamma}^{2}$, see Macosko (1994), vs shear rate, for both the Rolie-Poly and Leonov models, using the viscoelastic data in Table 1 . It can be seen that both models predict almost identical results; thus, minor differences might be expected, in terms of the magnitude of the shear-induced peaks, when using the Leonov or Rolie-Poly model.

Figure 9 shows the values of N1 at different locations along the mold cavity at the end of the filling phase. It clearly shows that $\mathrm{N} 1$ is maximum close to the middle of the cavity and decreases to zero towards the end of the cavity, due to the small deformation history the

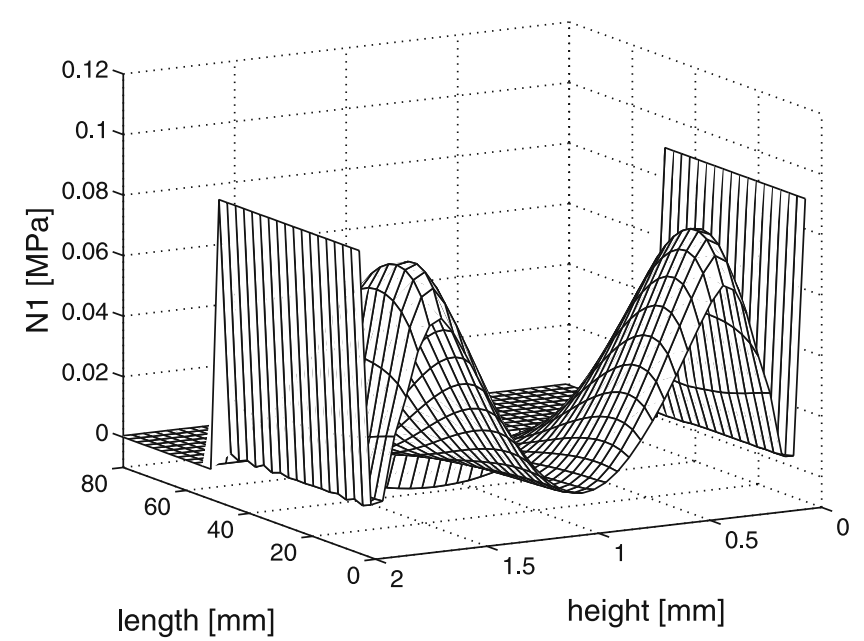

(end of polymer filling)

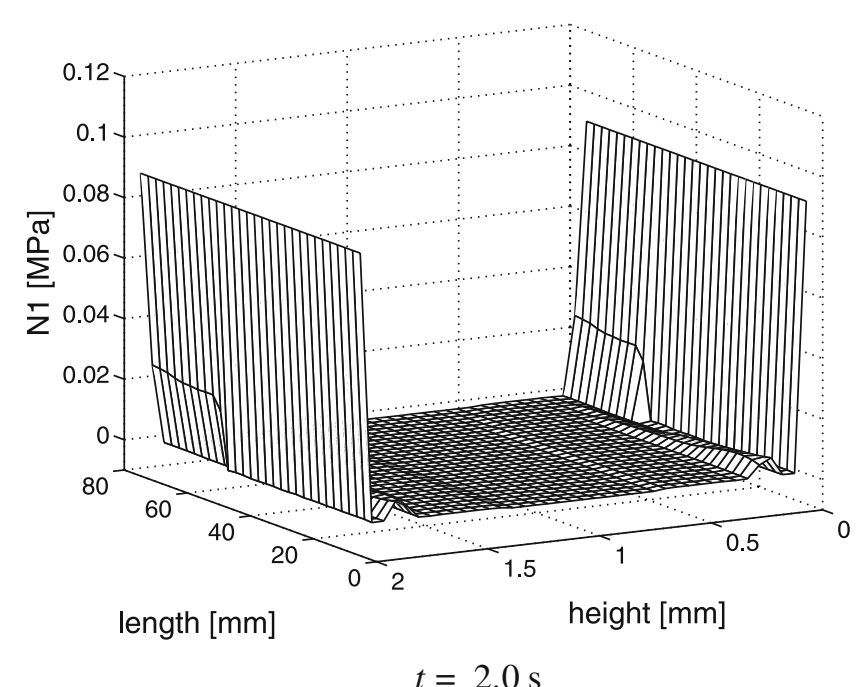

Fig. 11 Time evolution of N1 in the GAIM case material experiences there. Notice the double peak in the curve at he entrance of the cavity. We speculate that this peak is related to re-melting of the material during filling. Since we did not anticipate on this transient effect, we did not analyze it, i.e., store the full 3D thermal history. This effect is not new; a similar result was found by Douven (1991), see Fig. 6.11 in his thesis.

Figure 10 shows the evolution of N1 during the total injection molding cycle. After filling, the residual stresses are drastically reduced by fast relaxation at the characteristic high melt temperature, and their value remains only at the walls, where stress is frozen during filling. Upon packing, a third peak in between the shear-induced peak, developed during filling, and the center line develops. Although the strain rates are low during packing and holding, the relaxation times

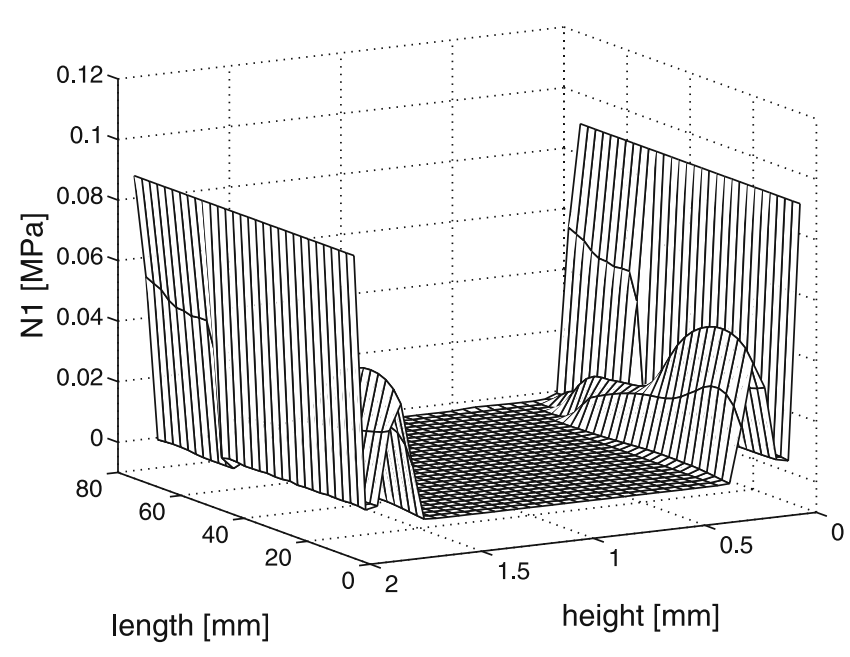

(during gas filling) 


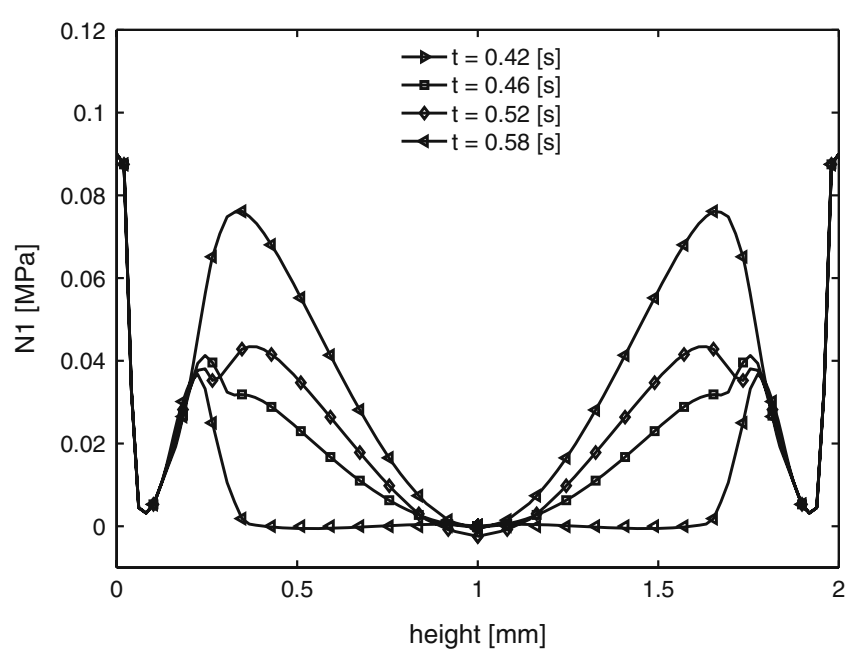

(a)

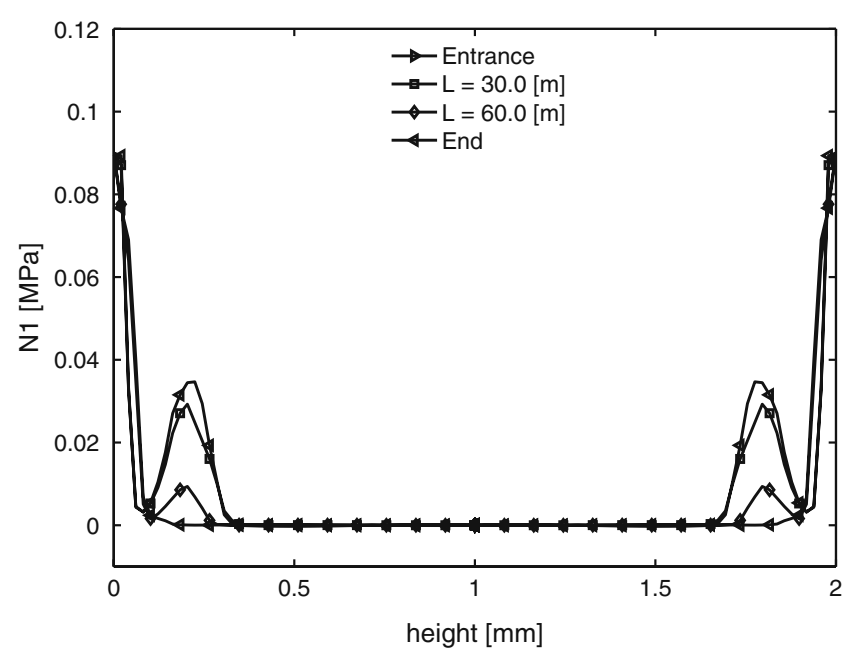

(b)

Fig. 12 a N1 profiles at $x=0.02 \mathrm{~m}$, depicting the transition from polymer filling to gas filling. $\mathbf{b} \mathrm{N} 1$ profiles along the cavity length after the completion of gas filling

increase due to cooling. They are clearly responsible for the development of significant orientation in these phases of the process, since a prerequisite for molecular orientation is sufficient strain rate: $\dot{\varepsilon}>1 /\left(\tau\left(T, M_{\mathrm{w}}\right)\right.$, with $\tau\left(T, M_{\mathrm{w}}\right)$ the temperature and molecular weightdependent relaxation times and sufficient strain $\varepsilon=\dot{\varepsilon} t>1$.

\section{Gas-assisted injection molding}

Figure 11 shows the evolution of N1 during the GAIM cycle. The transition from polymer filling to gas filling is marked by a sudden decrease in N1. The transition is illustrated in Fig. 12, where normal stress differences are plotted over the height of the channel for different times at a position close to the gate corresponding to $x_{1}=0.02 \mathrm{~m}$. At $t=0.42 \mathrm{~s}$, gas is injected and between $t=0.52 \mathrm{~s}$ and $t=0.58 \mathrm{~s}$, the gas front passes through $x_{1}=0.02 \mathrm{~m}$. Since the gas is only injected through the core of the part, significant relaxation occurs in the region of the shear-induced peaks. Only the molten material located in the core region is displaced. Thus, the deformation rates are drastically decreased, and since temperatures are still well above the glass transition temperature, the flow-induced stresses are allowed to relax until a new N1 profile is achieved; see Fig. 12a for $t=0.58 \mathrm{~s}$. Nevertheless, at the flow front, deformation of the material takes place as the molten polymer is pushed forward by the gas pressure. This is shown in Fig. 12b, in which normal stress differences, plotted at the end of the polymer filling phase for different positions along the cavity length close to the mold wall remain unchanged throughout the channel. When compared with the N1 results obtained with conventional injection molding, Fig. 9, there is an impressive reduction in flow-induced stresses. It can be seen that the height and width of the shear-induced peaks is significantly decreased by gas injection. Moreover, the packing and holding phases do not induce further orientation. This is due to the lower pressure level inside the cavity (and the fact that no further material is pushed into the cavity). Since the gas is able to exert pressure everywhere inside the cavity, the pressure level needed is lower (in this example, two orders of magnitude) than in the conventional case. As a consequence, in GAIM, the resulting frozen-in orientation and related flow-

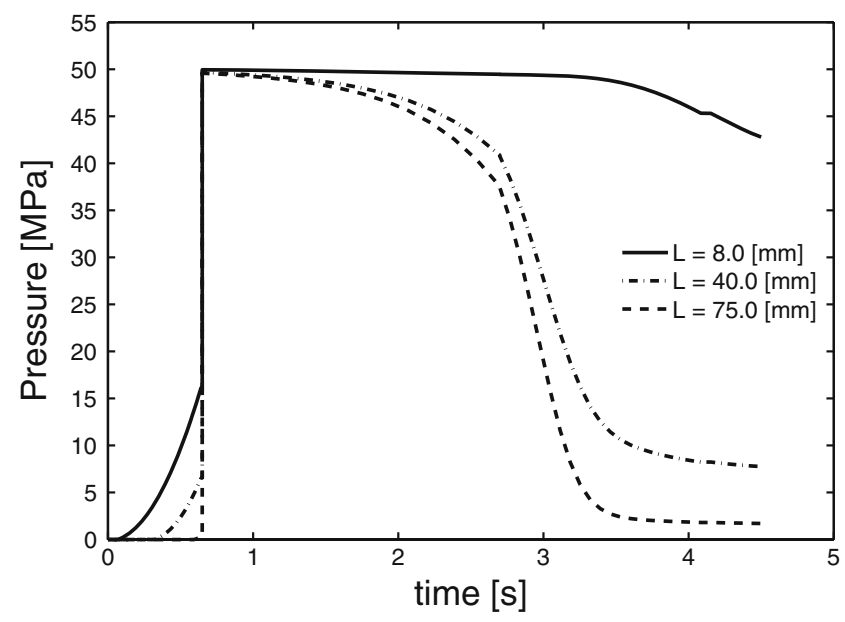

Fig. 13 Pressure history inside the cavity at different locations for the standard injection molding case 


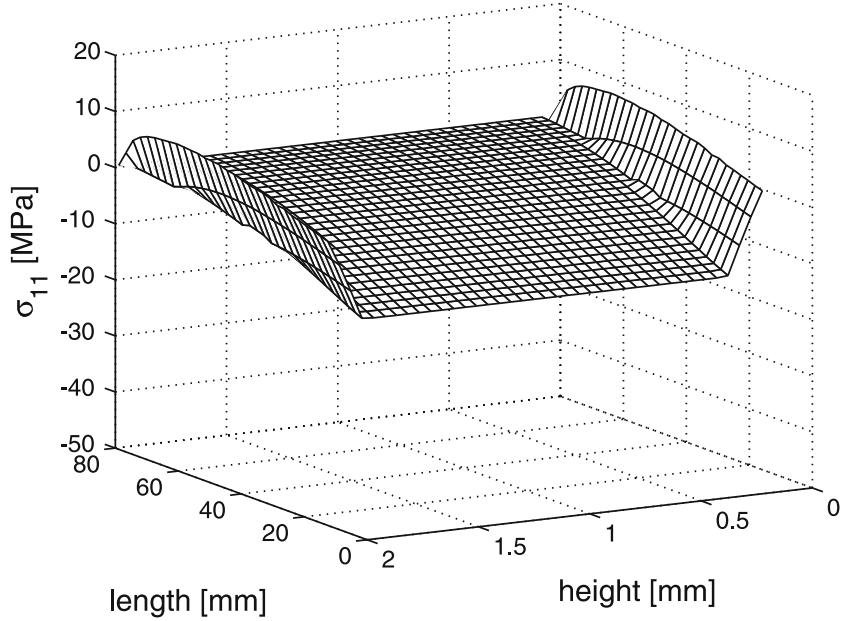

(end of filling)

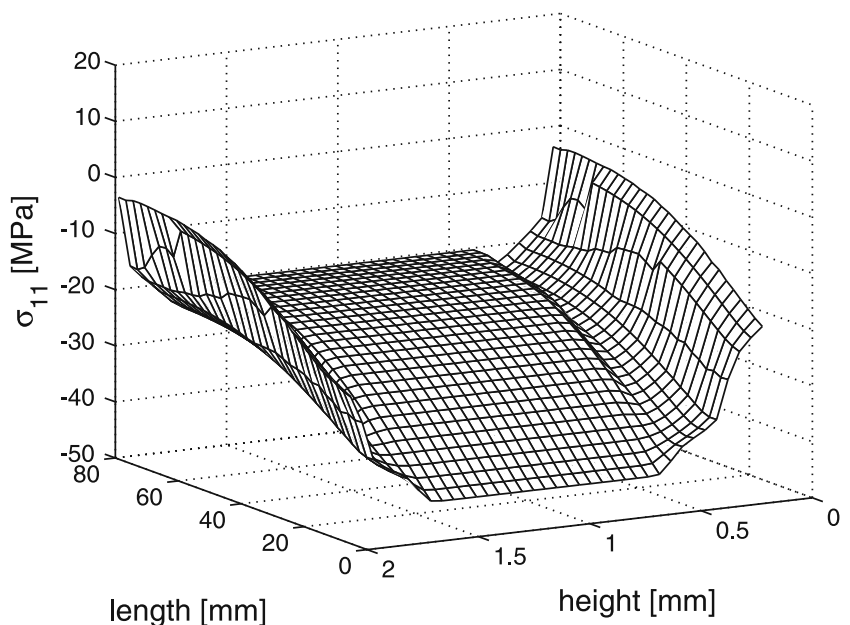

$t=2.4 \mathrm{~s}$

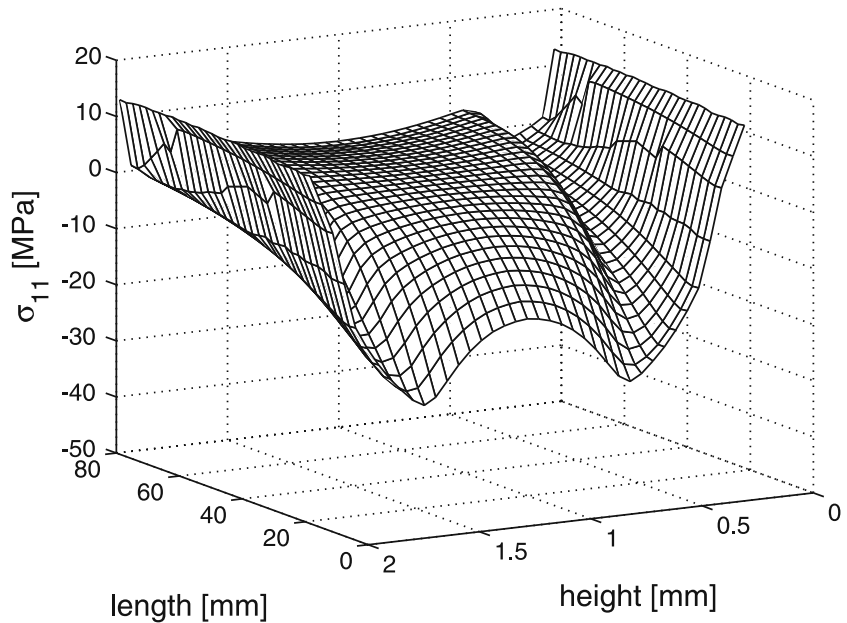

$t=4.4 \mathrm{~s}$

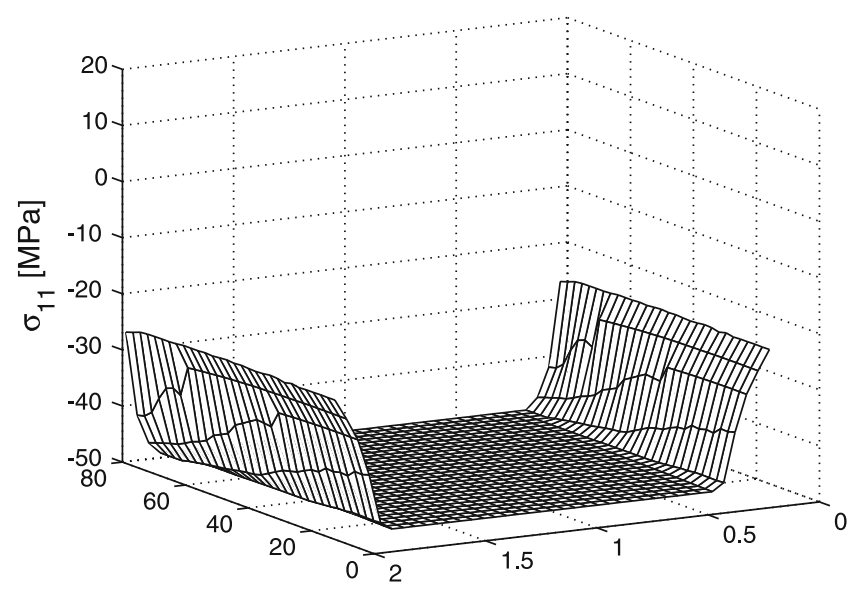

length [mm] height [mm]

$t=1.3 \mathrm{~s}$

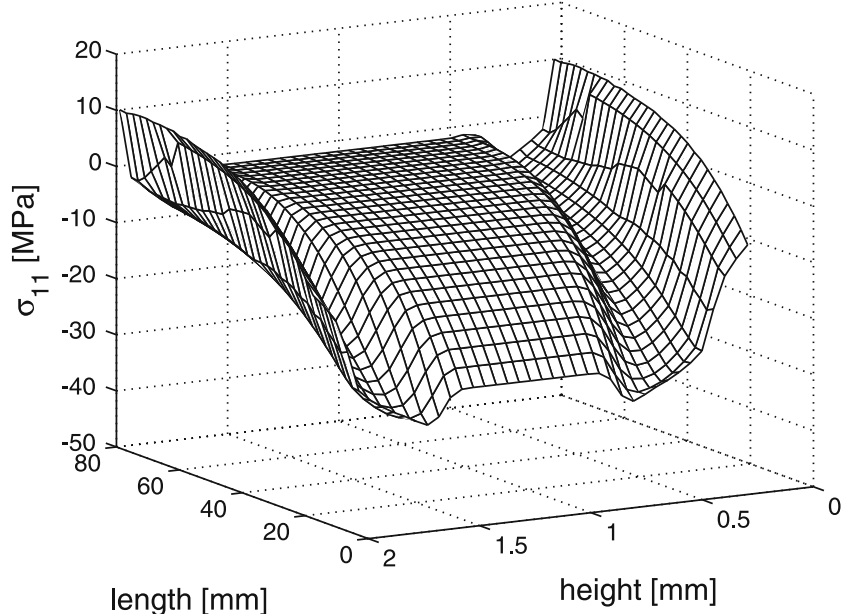

$t=3.3 \mathrm{~s}$

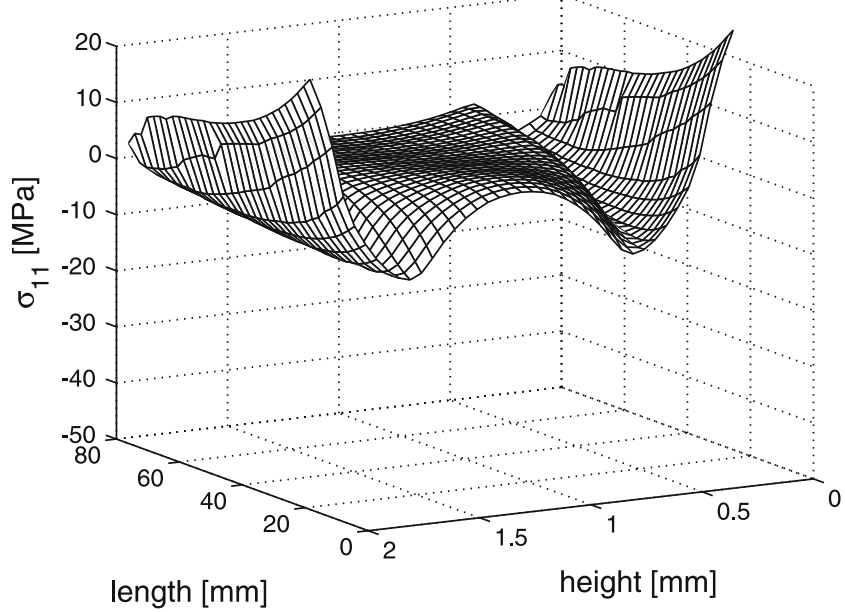

(upon ejection)

Fig. 14 Time evolution of thermally and pressured-induced stresses in the convention injection molding case 


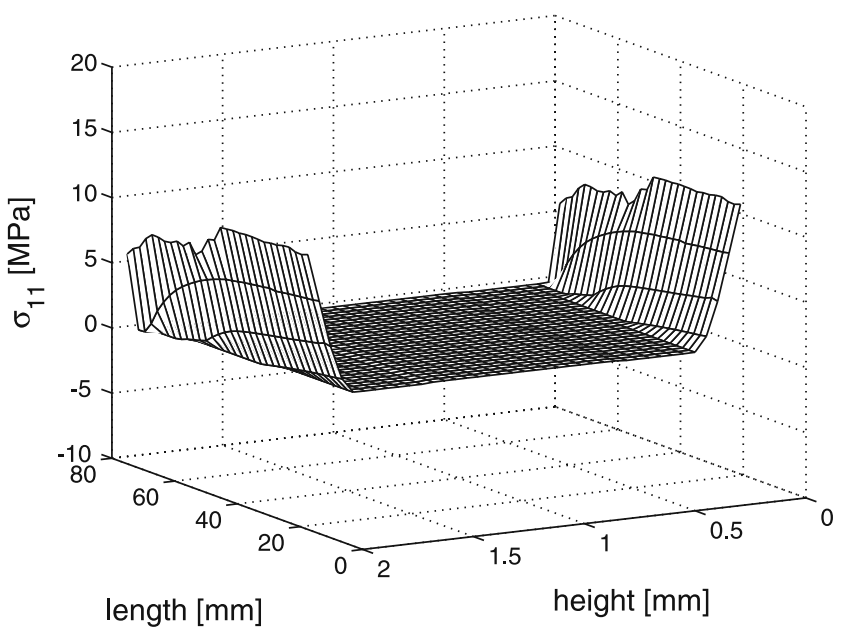

(end of filling)

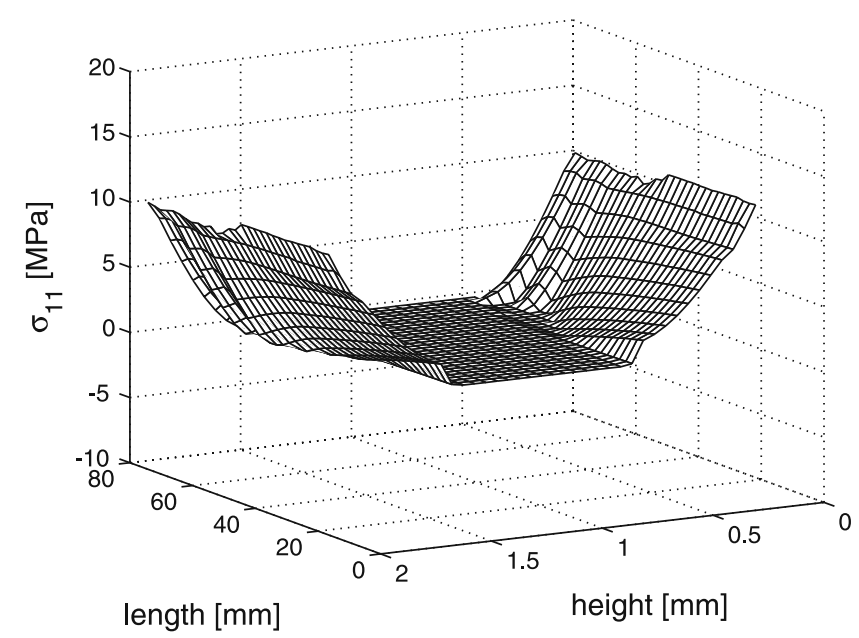

$t=1.8 \mathrm{~s}$

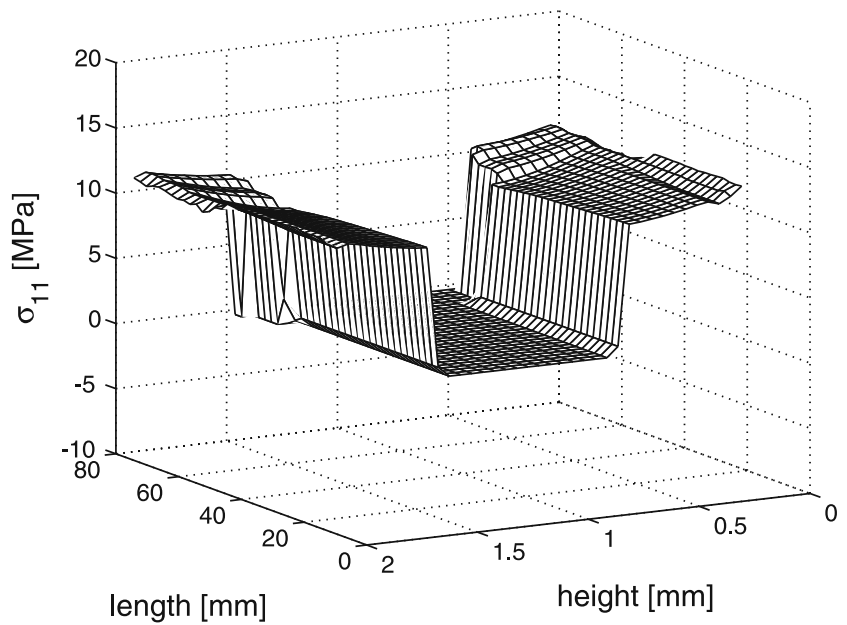

$t=3.5 \mathrm{~s}$

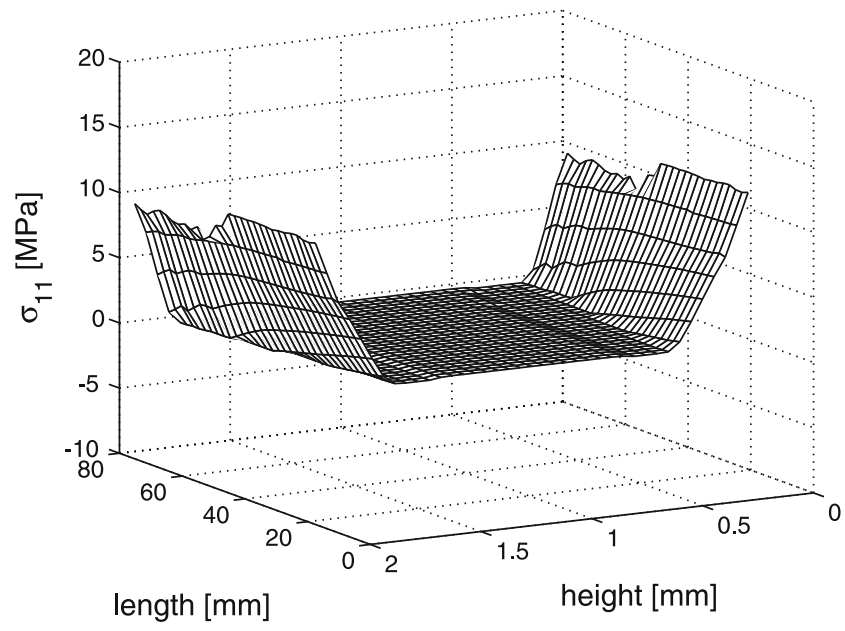

$t=1.4 \mathrm{~s}$

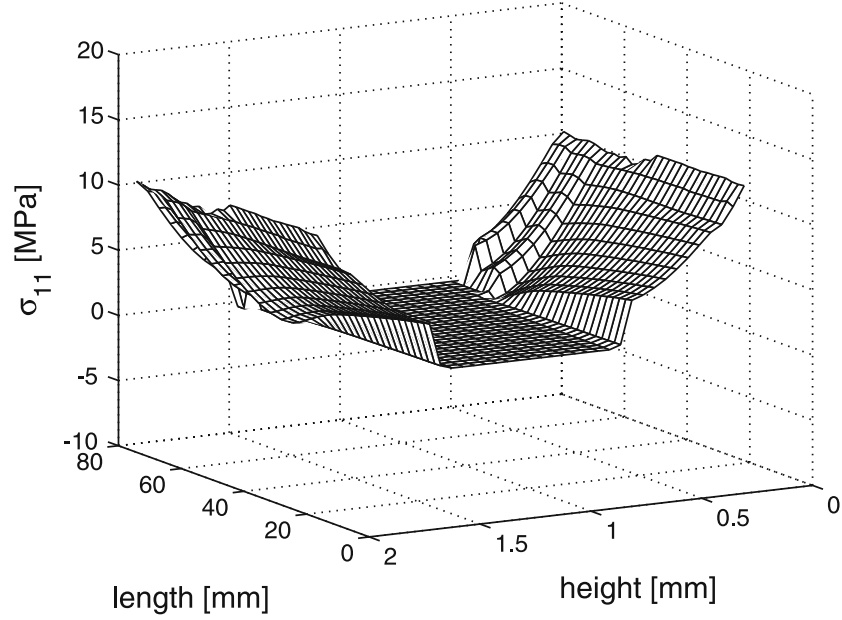

$t=2.0 \mathrm{~s}$

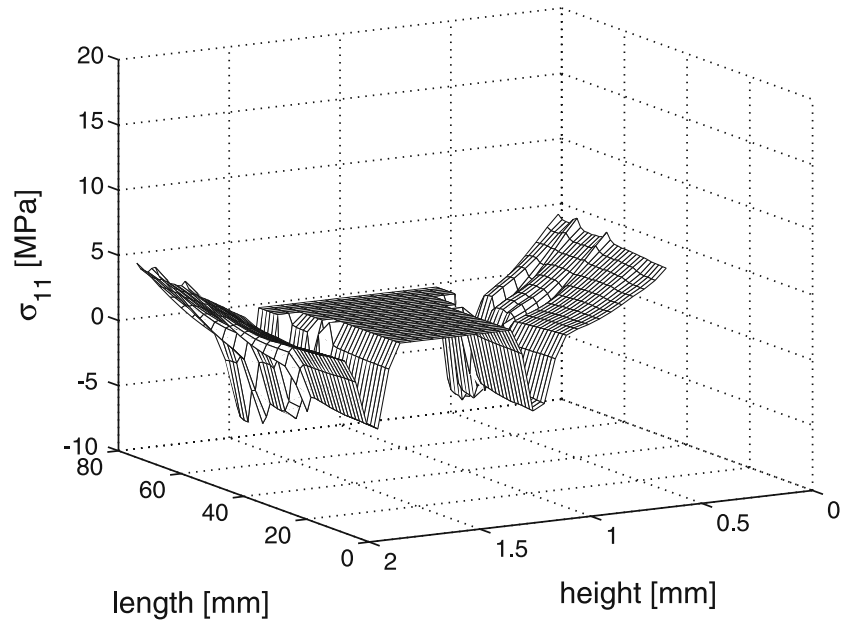

(upon ejection)

Fig. 15 Time evolution of thermally and pressured-induced stresses in the GAIM case 


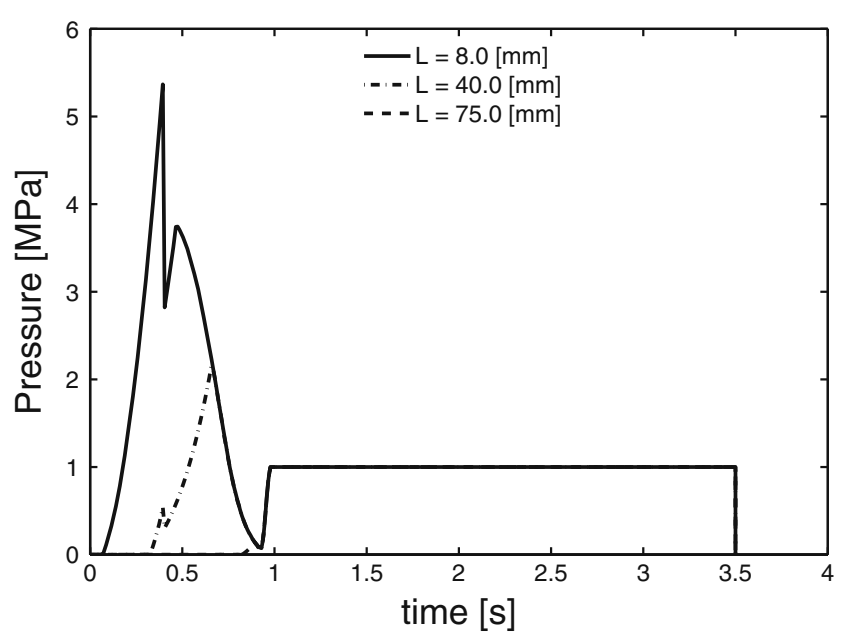

Fig. 16 Pressure history inside the cavity at different locations for the GAIM case

induced stresses are solely determined during the filling phase.

Thermally and pressure-induced residual stresses

\section{Conventional injection molding}

Figure 13 shows the calculated pressure history inside the cavity in three different positions, $x_{1}=0.008 \mathrm{~m}$, $x_{2}=0.04 \mathrm{~m}$, and $x_{3}=0.075 \mathrm{~m}$. The pressure decay is mostly determined by $\alpha$ and $\kappa$, and its evolution has a direct impact on the pressure-induced stresses. After the filling phase, $t_{f}=0.67 \mathrm{~s}$, the change to packing is marked by a steep rise in pressure. Figure 14 shows the evolution of the thermally and pressure-induced stress components. At the end of filling, only tensile stresses develop in the regions near to the mold walls, induced by the hampered thermal contraction while the pressure is maximum at the injection gate $L=0$ and zero at the end $L=0.08 \mathrm{~m}$. In the packing phase, the pressure increases to become uniform inside the mold cavity. Shrinkage upon cooling makes the pressure lower during the holding phase, and upon further material solidification, the pressure keeps on decaying. The pressure history is reflected in the stresses in the solidified layers compared to the bulk. Residual stresses become compressive in layers that solidify under a high pressure, and tensile in those solidified under a low pressure. At the end of the holding phase, the stresses in the solidified layers increase proportionally with the pressure relief.

\section{Gas-assisted injection molding}

Figure 16 shows the pressure history at three different positions along the cavity's length, $x_{1}=0.008 \mathrm{~m}, x_{2}=$ $0.04 \mathrm{~m}$, and $x_{3}=0.075 \mathrm{~m}$ for the GAIM case. The transition from polymer to gas injection can be seen by a decay and a subsequent growth of the pressure at $t_{f}=0.3 \mathrm{~s}$ at positions $x_{1}$ and $x_{2}$. The difference between the computed pressure histories for the conventional and GAIM cases immediately suggests that the residual stresses in GAIM should differ substantially. In GAIM, the end of filling occurs at a decaying pressure, since only the molten-liquid material in the core region is displaced, and the packing, and subsequently, holding, pressures are much lower. Also for the GAIM case, during the holding phase, the pressure inside the cavity

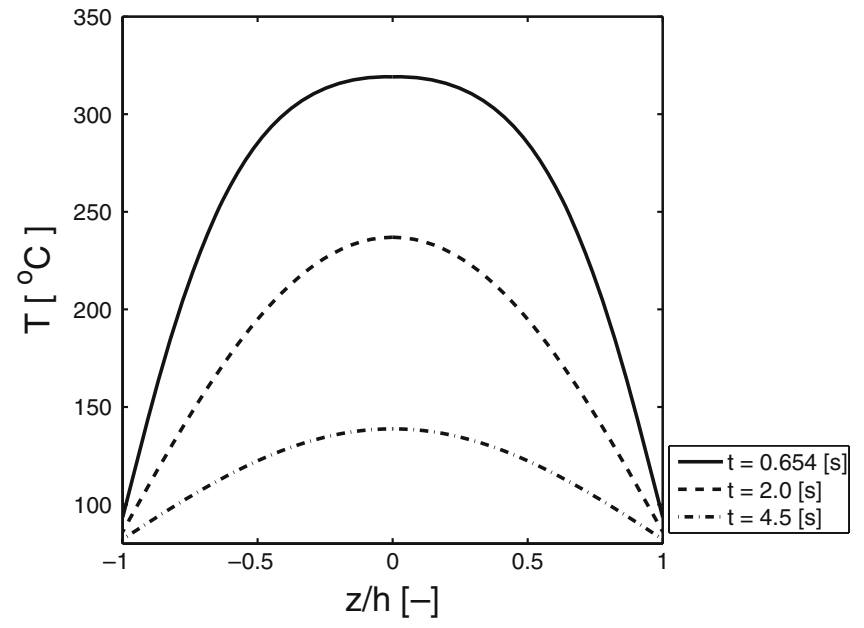

(a)

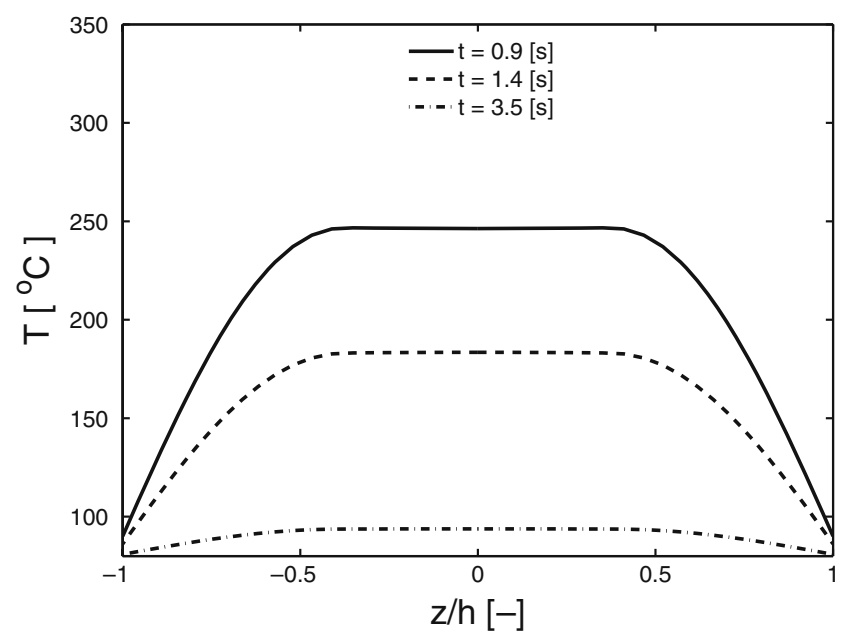

(b)

Fig. 17 Temperature history at $x_{2}=0.04 \mathrm{~m}$ for: a conventional injection molding; b GAIM 


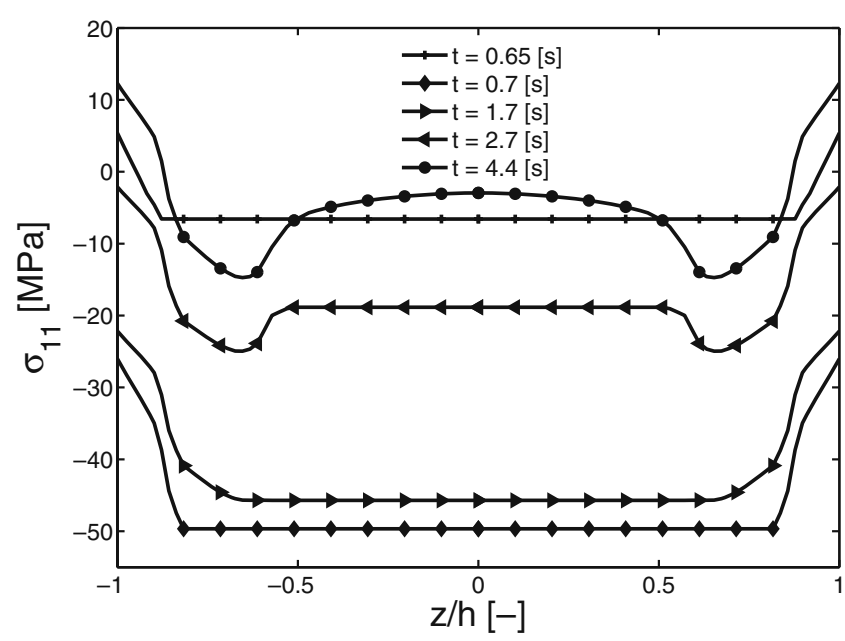

(a)

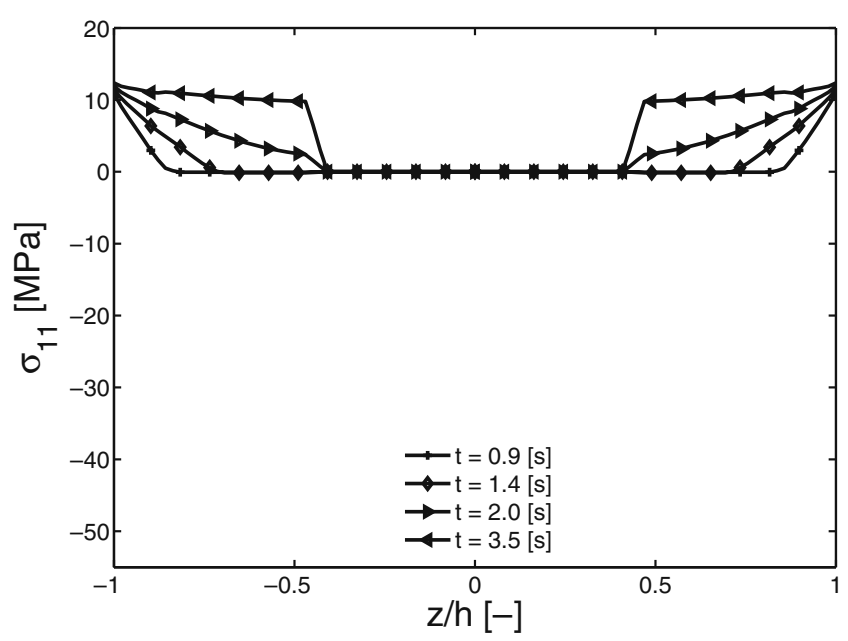

(b)

Fig. 18 Thermally and pressured-induced stresses at $x_{2}=0.04$ : a conventional injection molding; b GAIM

remains constant, and therefore, the polymer material solidifies at a constant pressure. Figure 15 shows the evolution of the thermally and pressure-induced $\sigma_{11}$ stress component. The profiles at the end of filling show a slightly higher tensile stress than in the conventional case. This can be related to the increased cooling in GAIM, which results from the absence of a molten core and a longer filling time (Fig. 16). In Fig. 17, temperature profiles are plotted in the middle of the part at the end of the respective filling phases for both conventional injection molding and GAIM. The increased cooling in the case of GAIM is clear. As a result of a shorter cooling time and a higher cooling rate, the thermal contraction prevented during filling is expected to be higher, thus inducing higher tensile

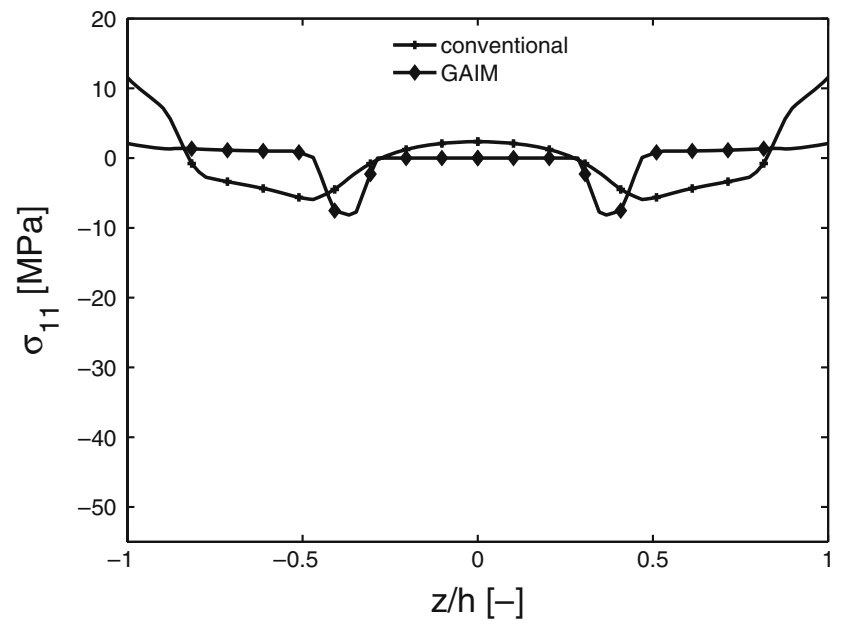

Fig. 19 Thermally and pressured-induced stresses at $x_{2}=0.04 \mathrm{~m}$ upon ejection stresses. As cooling proceeds, the tensile stresses propagate to reach the inner surface, resulting in a arc-type profile. Given the significant lower pressures, especially in the packing phase, the development of a compressive peak does not occur inside the mold. Close to the end of the slit, the stress profile shows some instabilities, which were found to be dependent on changes of the thickness of the solidified layer, which could not be made smoother via mesh refinement. Thus, the stress field is very sensitive to changes in the thickness of the previously solidified layer. Figure $18 \mathrm{a}$ and $\mathrm{b}$ give the evolution of the residual stress profiles at position $x_{2}$ in time, for conventional injection molding and GAIM. The final residual stress profiles obtained from the structural analysis are given in Fig. 19. It can be seen that, for GAIM, the tensile stresses at the wall are much lower compared to the conventional case.

\section{Conclusions}

A numerical study to assess the effect of GAIM on the development of residual stresses was conducted. Firstly, the evolution of flow-induced stresses in both conventional and GAIM was assessed. A compressible version of the Rolie-Poly model was proposed and applied for the computation of flow-induced stresses. Computational results show a significant decrease in the magnitude of stresses during the gas-filling phase. Moreover, it was found that the stress level did not evolve further during the packing and holding phases. Hence, the magnitude of flow-induced orientation and related stresses in GAIM is much lower than in the conventional case and is set during the filling phase only. 
Secondly, the development of thermally and pressureinduced stresses in GAIM was investigated. A thermoviscoelastic model was used to compute thermally and pressure-induced stresses. Computed stress profiles for GAIM during filling and holding phases exhibit slightly higher tensile stresses at the surface; however, the most noticeable difference is the absence of a compressive region, which is typical for conventional injection molded parts. This can be explained by the fact that the pressures for GAIM are much lower than those of conventional injection molding during the packing and holding phases. Knowing that, in injection molding, pressure-induced stresses overrule the contribution for the total residual stresses, it has been shown that GAIM can drastically change the residual stresses in injection molded parts. The final equilibrated stress profile shows that GAIM can significantly reduce tensile stresses at the walls. This is obviously of importance, since tensile stresses can initiate crazing and, thereafter, surface cracking. Our study supports that GAIM has a strong effect on the behavior of injection molding parts and their properties. It reduces the level of frozenin orientation and can therefore minimize the part's long-term dimensional changes and the anisotropy in physical properties. The simulations can be improved with constitutive models for density and heat conduction with better characterized material data. Analyses to assess the influence of processing conditions and of the part's geometry on computed stresses, which were beyond the scope of our study, are of interest to further exploit the GAIM characteristics to arrive at superior products with enhanced dimensional stability.

Open Access This article is distributed under the terms of the Creative Commons Attribution Noncommercial License which permits any noncommercial use, distribution, and reproduction in any medium, provided the original author(s) and source are credited.

\section{Appendix A}

Numerical integration of the Rolie-Poly model

To perform the numerical integration of the RoliePoly equation, Eq. 12, we use a second-order AdamsBashford explicit scheme. For the first two time steps, the numerical integration follows a first-order forward Euler method. Accordingly, the time marching scheme reads:

for time step $\leq 2$

$\overline{\boldsymbol{B}}_{e}^{n+1} \approx \overline{\boldsymbol{B}}_{e}^{n}+\left.\mathbf{f}\left(\mathbf{u}, \overline{\boldsymbol{B}}_{e}, \nabla \mathbf{u},(\nabla \mathbf{u})^{T}\right)\right|^{n} \triangle t$, for time step $>2$,

$$
\begin{aligned}
\overline{\boldsymbol{B}}_{e}{ }^{n+1} \approx \overline{\boldsymbol{B}}_{e}^{n}+( & \left.\frac{3}{2} \mathbf{f}\left(\mathbf{u}, \overline{\boldsymbol{B}}_{e}, \nabla \mathbf{u},(\nabla \mathbf{u})^{T}\right)\right|^{n} \\
& \left.-\left.\frac{1}{2} \mathbf{f}\left(\mathbf{u}, \overline{\boldsymbol{B}}_{e}, \nabla \mathbf{u},(\nabla \mathbf{u})^{T}\right)\right|^{n-1}\right) \triangle t .
\end{aligned}
$$

\section{Appendix B}

Incremental formulation of the thermo-viscoelastic model

We will now discretize the linear thermo-viscoelastic model, expressing it in an incremental formulation. At time $t=t_{n}$, the model, given by Eqs. 19 and 24, reads:

$$
\begin{aligned}
& \boldsymbol{\sigma}_{n}=-p_{n}^{h} \boldsymbol{I}+\sum_{j=1}^{m} \boldsymbol{\tau}_{j_{n}}^{d}, \\
& p_{n}^{h}=\int_{0}^{t_{n}}\left(\frac{\alpha}{\kappa} \dot{T}-\frac{1}{\kappa} \operatorname{tr}(\boldsymbol{D})\right) d s, \\
& \boldsymbol{\tau}_{j_{n}}^{d}=2 \int_{0}^{t_{n}} G_{j} e^{-\left(\xi_{n}-\xi(s)\right) / \tau_{i 0}} \dot{\varepsilon}^{d} d s .
\end{aligned}
$$

The subscript $n$ indicates the evaluation at time $t_{n}$, at which the model variables are fully determined. For the evaluation of the stress at the next step, $t_{n+1}$, it is required to know the temperature and strain fields. We introduce the following incremental variables for $T$ and $\xi$ :

$\Delta \xi_{n+1}=\xi_{n+1}-\xi_{n}, \quad \Delta T_{n+1}=T_{n+1}-T_{n}$.

It is assumed that $\xi$ and $T$ vary linearly between two discrete time steps, implying that $\dot{\varepsilon}$ and $\dot{T}$ are constant over each time increment. It yields

$\dot{T}=\frac{\Delta T_{n+1}}{\Delta t_{n+1}}, \quad \boldsymbol{\varepsilon}=\boldsymbol{D} \Delta t, \quad$ for $t \in\left[t_{n}, t_{n+1}\right]$,

Next, we discretize Eq. 19, and evaluate the integrals using the trapezium rule:

$p_{n+1}=p_{n}+\tilde{\beta} \Delta T_{n+1}-\tilde{K} \operatorname{tr}(\boldsymbol{D})$,

$\tilde{\beta}=\frac{1}{\Delta t_{n+1}} \int_{t_{n}}^{t_{n+1}} \frac{\alpha}{\kappa} d s$,

$\tilde{K}=\frac{1}{\Delta t_{n+1}} \int_{t_{n}}^{t_{n+1}} \frac{1}{\kappa} d s$. 
The discretization of Eq. 24, in combination with the trapezium rule, leads to:

$$
\begin{aligned}
& \sum_{j=1}^{m} \boldsymbol{\tau}_{j_{n+1}}^{d}=\sum_{j=1}^{m} \xi_{j_{n+1}} \boldsymbol{\tau}_{j_{n}}^{d}+2 \tilde{G} \Delta \boldsymbol{\varepsilon}_{n+1}^{d}, \\
& \tilde{G}=\sum_{j=1}^{m} \frac{G_{j 0}}{2}\left(1+\xi_{j_{n+1}}\right), \\
& \xi_{j_{n+1}}=\exp \left[-\frac{\Delta t_{n+1}}{2 \tau_{j 0}}\left(\frac{1}{a_{T_{n+1}}}+\frac{1}{a_{T_{n}}}\right)\right] .
\end{aligned}
$$

After extensive rewriting of Eq. 44 for $t=t_{n+1}$, the following discretized form for $\sigma$ is found:

$\boldsymbol{\sigma}_{n+1}=\tilde{\boldsymbol{\sigma}}+\tilde{K} \operatorname{tr}\left(\Delta \boldsymbol{\varepsilon}_{n+1}\right) \boldsymbol{I}+2 \tilde{G} \Delta \boldsymbol{\varepsilon}_{n+1}^{n}$,

where

$\tilde{\boldsymbol{\sigma}}=-\left(p_{n}+\tilde{\beta} \Delta T_{n+1}\right) \boldsymbol{I}+\sum_{j=1}^{m} \xi_{j_{n+1}} \boldsymbol{\tau}_{j_{n}}^{d}$.

The quantities $\tilde{\boldsymbol{\sigma}}, \tilde{K}$, and $\tilde{\beta}$ are evaluated when the state at $t_{n}$ is determined and the temperature history is known up until $t_{n+1}$. The stress components of the linear thermo-viscoelastic model are written with respect to the local base $O_{1}$, defined in Fig. 1. Due to assumption 5 and the knowledge of $\sigma_{22}$, the incremental strain component $\Delta \varepsilon_{22}$ can be eliminated from Eq. 55 :

$\Delta \varepsilon_{22}=\frac{\sigma_{22}-\tilde{\sigma_{22}}}{a}, \quad a=\frac{3 \tilde{K}+4 \tilde{G}}{3}$.

The final form of the discretized model reads:

$\sigma^{n+1}=\bar{a} \Delta \varepsilon_{11}+g$,

where

$\bar{a}=a-\frac{b^{2}}{a} \quad$ and $\quad g=\tilde{\sigma}_{11}+b / a\left(\sigma_{22}-\tilde{\sigma}_{22}\right)$,

with

$a=\frac{4 \tilde{G}+3 \tilde{K}}{3}, \quad b=\frac{-2 \tilde{G}+3 \tilde{K}}{3}$.

\section{References}

Amestoy P, Duff I (1989a) Memory management issues in sparse multifrontal methods on multiprocessors. Int J Supercomput Appl 7:64

Amestoy P, Duff I (1989b) Vectorization of a multiprocessor multifrontal code. Int J Supercomput Appl 3:41
Amestoy P, Puglisi C (2002) An unsymmetrical multifrontal lu factorization. SIAM J Matrix Anal Appl 24:553

Baaijens F (1991) Calculation of residual stresses in injection molded products. Rheol Acta 30:284-299

Bird R, Armstrong R, Hassager O (1987) Dynamics of polymer liquids, vol 1. Wiley-Interscience, New York

Bogaerds A, Hulsen M, Peters G, Baaijens F (2002) Stability analysis of injection molding flows. J Rheol 48:765-785

Brooks A, Huges T (1982) Streamline upwind/petrov-galerkin formulation for convection dominated flows with particular emphasis on the incompressible navier-stokes equations. Comput Methods Appl Mech Eng 32:199-259

Caspers L (1995) Vip, an integral approach to the simulation of injection moulding. $\mathrm{PhD}$ thesis, Eindhoven University of Technology, Eindhoven

Doi M, Edwards S (1986) The theory of polymer dynamics. Claredon, Oxford

Douven L (1991) Towards the computation of properties of injection moulded products. PhD thesis, Eindhoven University of Technology, Eindhoven

Douven L, Baaijens F, Meijer H (1995) The computation of properties of injection-moulded products. Prog Polym Sci 20:403-457

Grillet A, Bogaerds A, Peters G, Baaijens F, Bulters M (2002) Numerical analysis of flow mark surface defects in injection molding flow. J Rheol 46:651-669

Haagh G, Van de Vosse F (1998) Simulation of threedimensional polymer mould filling processes using a pseudoconcentration method. Int J Numer Methods Fluids 28: 1355-1369

Haagh G, Van de Vosse F (2001) A 3-d finite element model for gas-assisted injection molding: simulations and experiments. Polym Eng Sci 41:449-465

Hansen D, Bernier G (1972) Thermal conductivity of polyethylene:the effects of crystal size, density and orientation on the thermal conductivity. Polym Eng Sci 12:204

Hastenberg C, Wildervank P, Leeden A (1998) The measurement of thermal stresses distributions along the flow path in injection-molded flat plates. Polym Eng Sci 32:506-515

Hulsen A, Fattal R, Kupferman R (2005) Flow of viscoelastic fluids past a cylinder at high weissenberg number: stabilized simulations using matrix logatithms. J Non-Newton Fluid Mech 127:27-39

Kamal M, Tan V (1979) Orientation in injection molded polystyrene. Polym Eng Sci 19:558-563

Kamal M, Fook RA, H AJR (1988) Residual thermal stresses in injection moldings of thermoplastics: a theoretical and experimental approach. Polym Eng Sci 42:1098-1114

Kennedy PK (2008) Pratical and scientific aspects of injection molding simulation. PhD thesis, Eindhoven University of Technology, Eindhoven

Klompen E (2005) Mechanical properties of solid polymers: constitutive modelling of long and short term behaviour. $\mathrm{PhD}$ thesis, Eindhoven University of Technology, Eindhoven

Li C, Shin J, AI I (2004) Primary and secondary gas penetration during gas-assisted injection molding. Part 2: simulation and experiment. Polym Eng Sci 44:992-1002

Likhtman A, Graham R (2003) Simple constitutive equation for linear polymer melts derived from molecular theory: Rolie-poly equation. J Non-Newton Fluid Mech 114: $1-12$

Macosko C (1994) Rheology: principles, measurements, and applications. Wiley-VCH, New York

Mandell J, Smith K, Huang D (1981) Effects of residual stress and orientation on the fatigue of injection molded polysulfone. Polym Eng Sci 21:1173-1180 
Mavridis H, Hrymak A, Vlachopoulos J (1988) The effect of fountain flow on molecular orientation in injection molding. J Rheol 32:639-663

Meijer H (1997) Processing of polymers. In: Meijer H (ed) Material science and technology, vol 18, chap 1. VCH, Verlagsgesellschaft $\mathrm{mbH}$, Weinheim

Parvez M, Ong N, Lam Y, Tor S (2002) Gas-assisted injection molding: the effects of process variables and gas channel geometry. J Mater Process Technol 121:27-35

Polynkin A, Pittman J, Sienz J (2005) 3d simulation of gas assisted injection molding analysis of primary and secondary gas penetration and comparison with experimental results. Int Polym Process 20:191-201

Saad Y, Schultz M (1992) Gmres: a generalized minimal residual algorithm for solving nonsymetric linear systems. SIAM J Matrix Anal 13:121-137

Simo J (1987) On a fully three-dimensional finite-strain viscoelastic damage model: formulation and computational aspects. Comput Methods Appl Mech Engrg 60:153-173

Souheng Wu EI (1989) Chain structure and entanglement. J Polym Sci, Part B: Polym Phys 27:723-741
Struik L (1978) Orietation effects and cooling stresses in amorphous polymers. Polym Eng Sci 18:799-811

Tadmor Z (1974) Molecular orientation in injection molding. J Appl Polym Sci 18:1753-1772

Thompson E (1986) Use of pseudo-concentrations to follow creeping viscous flows during transient analysis. Int J Numer Methods Fluids 6:749-761

van den Brule B, O'Brien S (1990) Anisotropic conduction of heat in a flowing polymeric material. Rheol Acta 29: 580-587

van Krevelen D (1990) Properties of polymers. Elsevier Science B.V., Amsterdam

Wimberger-Friedl R, Bruin J, Schoo F (2003) Residual birrefringence in modified polycarbonates. Polym Eng Sci 43: $62-70$

Zoetelief W, Douven L, Housz J (1996) Residual thermal stresses in injection molded products. Polym Eng Sci 36:1886-1896

Zoller P (1982) A study of the pressure-volume-temperature relationships of four related amorphous polymers: polycarbonate, polyarylate, phenoxy and polysulfone. Polym Sci 20:1453-1464 